\title{
Convergence of Mimetic Finite Difference Method for Diffusion Problems on Polyhedral Meshes
}

\author{
Franco Brezzi* Konstantin Lipnikov ${ }^{\dagger \ddagger}$ Mikhail Shashkov $^{\dagger}$
}

August 26, 2004

\begin{abstract}
The stability and convergence properties of the mimetic finite difference method for diffusion-type problems on polyhedral meshes are analyzed. The optimal convergence rates for the scalar and vector variables in the mixed formulation of the problem are proved.
\end{abstract}

\section{Introduction}

The main goal of this paper is to establish the convergence of mimetic discretizations of the firstorder system that describes linear stationary diffusion on general polyhedral meshes. The main idea of the mimetic finite difference (MFD) method is to mimic the underlying properties of the original continuum differential operators, e.g. conservation laws, solution symmetries, and the fundamental identities and theorems of vector and tensor calculus. For the linear diffusion problem, this means that the mimetic discretizations mimic the Gauss divergence theorem needed for the local mass conservation, the symmetry between the continuous gradient and divergence operators needed for proving symmetry and positivity of the resulting discrete operator, and the null spaces of the involved operators needed for stability of the discretizations.

The MFD method has been successfully employed for solving problems of continuum mechanics [18], electromagnetics [13], gas dynamics [7], and linear diffusion on simplicial and quadrilateral meshes in both the Cartesian and polar coordinates $[14,12,19,16]$. Recent advances in extending the mimetic discretizations to general polygonal meshes [15] have inspired us for developing the rigorous convergence theory for general polygonal and polyhedral meshes.

The polyhedral element appear naturally in reservoir models simulating thinning or tapering out ("pinching out") of geological layers. The pinch-outs are modelled with mixed types of mesh elements, pentahedrons, prisms and tetrahedrons which are frequently obtained by collapsing some of the elements in a structured hexahedral or prismatic mesh.

Other sources of polyhedral meshes are the adaptive mesh refinement methods. A necessity to have a conformal mesh results in an abundant mesh refinement, e.g. in the methods using

*Università di Pavia, Department of Mathematics, Pavia, Italy, brezzi @imati.cnr. it

${ }^{\dagger}$ Los Alamos National Laboratory, Theoretical Division, MS B284, Los Alamos, NM, 87545, \{lipnikov, shashkov\}@lanl.gov

${ }^{\ddagger}$ Corresponding author 
the red-green refinement strategy. However, the locally refined mesh may be considered as the conformal polyhedral mesh with degenerate elements (for instance, when the angle between two faces is zero). If we know how to discretize a problem on a general polyhedral mesh, the superfluous mesh refinements can be avoided. A similar argument can be applied to non-matching meshes which frequently may be treated as conformal polyhedral meshes with degenerate elements. This is the way followed for instance in [15] for 2D meshes.

Allowing arbitrary shape for a mesh element provides greater flexibility in the mesh generation process, especially in the regions where the geometry is extremely complex. Even in the case of an unstructured hexahedral mesh, it may be beneficial to split the curvilinear faces into triangles in order to use more accurate discretization methods and to get a smaller number of unknowns relative to a tetrahedral partition. It is obvious that by splitting each face of a hexagon into 4 triangles we get a 24 -face polyhedron which is frequently non-convex.

Some of the simulations in the fluid dynamics indicate that the polyhedral meshes may lead to superior convergence rates and accuracy relative to tetrahedral meshes. We refer readers to the CD adapco Group webcite [www.cd-adapco.com/news/18/newsdev.htm] for more detail. The polyhedral meshes are also used in a number of radiation-hydrodynamics applications [20, 21, 6]. For instance, one of the approaches to increase robustness of arbitrary Lagrangian-Eulerian simulations is to allow a change of the mesh connectivity which obviously leads to general polyhedral meshes.

The diffusion-type (elliptic) problems appear in many applications, for instance the temperature equation in heat diffusion or the pressure equation in flow problems. The necessity to solve such problems arises in numerical methods for radiation transport coupled with hydrodynamics, mesh smoothing algorithms, etc. In this paper we consider a diffusion problem formulated as a system of two first order equations, which is suitable for deriving locally conservative discretizations.

The mimetic discretizations have demonstrated excellent robustness and accuracy in simulations; however, a rigorous convergence proof has been always lacking. The original approach to prove the convergence of these discretizations has been based on establishing the relationship between the MFD and mixed finite element methods [2,3] which is certainly not enough for many interesting applications. In this paper, we developed a novel technique for proving convergence estimates which may be applied to the case of meshes consisting of arbitrary types of elements e.g., tetrahedrons, pyramids, hexahedrons, degenerate polyhedrons, etc. The restrictions imposed in Section 2 on the polyhedron shape allow still extremely complex elements which cover certainly the majority of meshes used in applications.

The paper contents is as follows. In Section 2, we describe the problem under consideration and the class of polyhedral meshes used in the convergence analysis. In Section 3, we formulate the mimetic finite difference method. In Section 4, we prove the stability result. In Section 5 , we prove the convergence of mimetic discretizations. One of the key elements used in our technique, the lift property, is discussed in detail in Appendix A. 


\section{The assumptions on the problem and on the mesh}

Let us consider a model elliptic boundary value problem:

$$
\begin{aligned}
& \operatorname{div} \mathbf{F}=b \\
& \mathbf{F}=-\mathbf{K} \operatorname{grad} p .
\end{aligned}
$$

Here $p$ denotes a scalar function that we refer to as the pressure, $\mathbf{F}$ denotes a vector function that we refer to as the velocity, $\mathbf{K}$ denotes a full symmetric tensor, and $b$ denotes a source function. The problem is posed in a bounded polyhedral domain $\Omega \subset \mathbb{R}^{3}$, and is subject to appropriate boundary conditions on $\partial \Omega$. For simplicity, we assume that the homogeneous Dirichlet boundary conditions are imposed on $\partial \Omega$. We assume also that $\mathbf{K}$ satisfies the following regularity and ellipticity property.

$\mathbf{P 1}$ (Regularity and ellipticity of $\mathbf{K}$ ). Every component of $\mathbf{K}$ is in $W_{\infty}^{1}(\Omega)$ and $\mathbf{K}$ is strongly elliptic, implying that there exist two constants $\kappa_{*}$ and $\kappa^{*}$ such that

$$
\kappa_{*}\|\mathbf{v}\|^{2} \leq \mathbf{v}^{T} \mathbf{K}(\mathbf{x}) \mathbf{v} \leq \kappa^{*}\|\mathbf{v}\| \quad \forall \mathbf{v} \in \mathbb{R}^{3} \quad \forall \mathbf{x} \in \Omega
$$

Let $\mathcal{T}_{h}$ be a non-overlapping conformal partition of $\Omega$ into polyhedral elements $E$. For every element $E$ we denote by $|E|$ its volume and by $h_{E}$ its diameter. Similarly, for each face $e$ we denote by $|e|$ its area and for every edge $\ell$ we denote by $|\ell|$ its length. We shall use $\partial E$ either for the boundary of $E$ or the union of element faces, according with convenience of the presentation. We also set as usual

$$
h=\sup _{E} h_{E}
$$

The elements $E$ are assumed to be closed simply-connected polyhedrons, rather general in shape (see for instance Figure 2). We need however some basic assumptions of shape regularity. As we shall see, the assumptions are sometimes formally complicated, but they will hold for practically all partitions which is not totally unreasonable.

M1 (Assumptions on the domain $\Omega$ ). We assume that $\Omega$ is a polyhedron with a Lipschitz continuous boundary.

M2 (Number of faces and edges). We assume that we have two positive integers $N_{e}$ and $N_{\ell}$ such that every element $E$ has at most $N_{e}$ faces, and each face $e$ has at most $N_{\ell}$ edges.

M3 (Volumes, areas, and lengths). We assume that there exist three positive constants $v_{*}, a_{*}$ and $l_{*}$ (for volume, area and length, respectively) such that for every element $E$ we have

$$
v_{*} h_{E}^{3} \leq|E|, \quad a_{*} h_{E}^{2} \leq|e|, \quad l_{*} h_{E} \leq|\ell|
$$

for all faces $e$ and edges $\ell$ of $E$.

M4 (Star-shaped faces). We assume that the mesh faces are flat and that there exists a positive number $\gamma_{*}$ such that: For each element $E$ and for each face $e \in \partial E$ there exists a point $M_{e} \in e$ such that $e$ is star-shaped with respect to every point in the disk of center $M_{e}$ and radius $\gamma_{*} h_{E}$. 
We recall that $e$ is star-shaped with respect to a point $P \in e$ if every straight ray exiting from $P$ (in the plane of $e$ ) intersects $\partial e$ only once. In what follows we shall often use the notation

$$
\rho_{*}=\gamma_{*} h_{E}
$$

which is illustrated in Fig. 1.

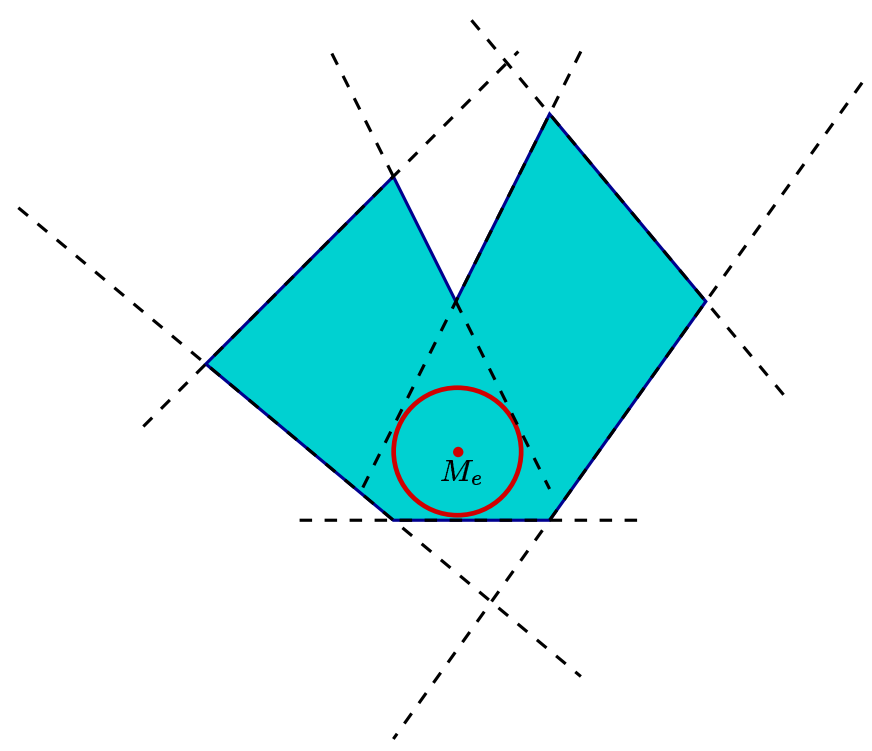

Figure 1: A star-shaped face, with the circle of radius $\rho_{*}$ centered at $M_{e}$.

M5 (The pyramid property). With the notation of assumption M4, we further assume that for every $E \in \mathcal{T}_{h}$, and for every $e \in \partial E$, there exists a pyramid $P_{E}^{e}$ contained in $E$ such that its base equals to $e$, its height equals to $\gamma_{*} h_{E}$ and the projection of its vertex onto $e$ is $M_{e}$.

M6 (Star-shaped elements). We assume that there exists a positive number $\tau_{*}$ such that: For each element $E$ there exists a point $M_{E} \in E$ such that $E$ is star-shaped with respect to every point in the sphere of center $M_{E}$ and radius $\tau_{*} h_{E}$.

As before, we say that $E$ is star-shaped with respect to a point $P \in E$ if every straight ray exiting from $P$ intersects $\partial E$ only once.

\section{Mimetic finite difference method}

Let us introduce an operator $\mathcal{G}, \mathcal{G} p=-\operatorname{Kgrad} p$, which we refer to as the flux operator. Furthermore, we introduce the following scalar products:

$$
(\mathbf{F}, \mathbf{G})_{X}=\int_{\Omega} \mathbf{F} \cdot \mathbf{K}^{-1} \mathbf{G} \mathrm{d} V
$$

and

$$
(p, q)_{Q}=\int_{\Omega} p q \mathrm{~d} V
$$


in the space $X$ of velocities and in the space $Q$ of pressures, respectively. Using the above notation, we may rewrite the Green formula

$$
\int_{\Omega} \mathbf{F} \cdot\left(\mathbf{K}^{-1} \mathcal{G} p\right) \mathrm{d} V=\int_{\Omega} p \operatorname{div} \mathbf{F} \mathrm{d} V
$$

in the equivalent form:

$$
(\mathbf{F}, \mathcal{G} p)_{X}=(p, \operatorname{div} \mathbf{F})_{Q}
$$

The last expression clearly states that the flux and divergence operators are adjoint to each other:

$$
\mathcal{G}=\operatorname{div}^{*} \text {. }
$$

The mimetic finite difference (MFD) method produces discretizations of these operators which are adjoint to each other with respect to scalar products in the discrete velocity and pressure spaces.

The first step of the MFD method is to specify the degrees of freedom for physical variables $p$ and $\mathbf{F}$ and their location.

We consider the space $Q^{d}$ of discrete pressures that are constant on each polyhedron $E$. For $\mathbf{q} \in Q^{d}$ we shall denote by $q_{E}$ (or by $(\mathbf{q})_{E}$ ) its (constant) value on $E$. The dimension $N_{Q}$ of $Q^{d}$ will obviously equal the number of polyhedrons in $\mathcal{T}_{h}$. In what follows, we shall denote by $Q^{d}$ either the vector space $\mathbb{R}^{N_{Q}}$ or the space of piecewise constant functions, according with convenience. The identification is obvious and no confusion should arise.

The definition of the space of discrete velocities will require some additional considerations. To every element $E$ in $\mathcal{T}_{h}$ and to every face $e$ of $E$ we associate a number $F_{E}^{e}$ and the vector field $F_{E}^{e} \mathbf{n}_{E}^{e}$ where $\mathbf{n}_{E}^{e}$ is the unit normal to $e$ that points outside of $E$. We clearly make the continuity assumption that for each face $e$ shared by two polyhedra $E_{1}$ and $E_{2}$, we have

$$
F_{E_{1}}^{e}=-F_{E_{2}}^{e}
$$

We denote the vector space of face-based velocity unknowns by $X^{d}$. The number $N_{X}$ of our discrete velocity unknowns will then, in our theory, be equal to the number of boundary faces plus twice the number of internal faces. In our theoretical discussion, we shall consider $X^{d}$ as the subspace of $\mathbb{R}^{N_{X}}$ which verifies (3.4).

For a discrete velocity field $\mathbf{G}$ we will denote by $\mathbf{G}_{E}$ its restriction to the boundary of $E$, and by $G_{E}^{e}$ (or by $\left(\mathbf{G}_{E}\right)^{e}$ ) the restriction of $\mathbf{G}_{E} \cdot \mathbf{n}_{E}$ to a face $e$ belonging to the boundary of $E$. It will sometimes be convenient to use the notation

$$
X_{E}^{d}:=\left\{\text { restrictions of } X^{d} \text { to the element } E\right\}
$$

It is clear that, in practice, condition (3.4) will make the number of true independent unknowns equal the total number of mesh faces. This means that, in a computer program, we shall prescribe one direction for the normal to each internal face $e$, and assign a single unknown $G^{e}$ to each face, assuming that each of the two $G_{E}^{e}$ coincides either with $G^{e}$ (when the outward normal $\mathbf{n}_{E}$ on $e$ coincides with the prescribed direction) or with $-G^{e}$ (otherwise).

To summarize, one pressure unknown is defined on each polyhedron and the discrete velocities are defined as face-based normal components. Once we got the degrees of freedom in $Q^{d}$ and in $X^{d}$, we can define interpolation operators from the spaces of smooth enough scalar and 

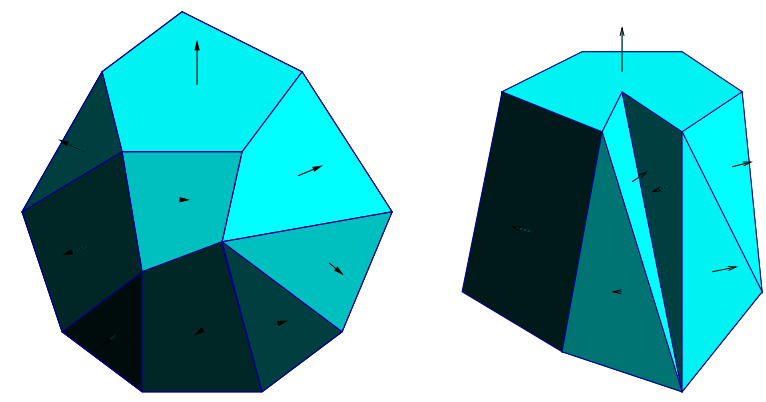

Figure 2: Two possible elements and the normal to their faces.

vector-valued functions to the discrete spaces $Q^{d}$ and $X^{d}$, respectively. To every function $\mathrm{q}$ in $L^{1}(\Omega)$ we associate the element $\mathbf{q}^{I} \in Q^{d}$ defined by

$$
\left(\mathbf{q}^{I}\right)_{E}:=\frac{1}{|E|} \int_{E} q \mathrm{~d} V \quad \forall E \in \mathcal{T}_{h} .
$$

Similarly, for every vector-valued function $\mathbf{G} \in\left(L_{s}(\Omega)\right)^{3}, s>2$, with $\operatorname{div} \mathbf{G} \in L^{2}(\Omega)$, we define $\mathbf{G}^{I} \in X^{d}$ by

$$
\left(\mathbf{G}_{E}^{I}\right)^{e}:=\frac{1}{|e|} \int_{e} \mathbf{G} \cdot \mathbf{n}_{E} \mathrm{~d} S \quad \forall E \in \mathcal{T}_{h} \quad \forall e \in \partial E .
$$

In the next section, we shall prove that this interpolation operator is well defined and uniformly bounded. In what follows, we shall use bold capital letters either for vectors from $X^{d}$ or for continuous vector functions, according with convenience of the presentation and leaving no room for confusion.

The second step of the MFD method is to equip the spaces of discrete pressures and velocities with scalar products. The scalar product on the vector space $Q^{d}$ is given by

$$
[\mathbf{p}, \mathbf{q}]_{Q^{d}}=\sum_{E \in \mathcal{T}_{h}} p_{E} q_{E}|E| \quad \forall \mathbf{p}, \mathbf{q} \in Q^{d} .
$$

In order to define the scalar product in $X^{d}$, we first define a scalar product $[\mathbf{F}, \mathbf{G}]_{E}$ for every element $E \in \mathcal{T}_{h}$ in the following way. Let $e_{1}, e_{2}, \ldots, e_{k_{E}}$ be a numbering of the faces of $E$ (where $k_{E}$ is clearly the total number of faces). We assume that we are given (for each $E$ ) a symmetric positive definite $k_{E} \times k_{E}$ matrix $M_{E} \equiv\left\{M_{E, i, j}\right\}$, and we set

$$
[\mathbf{F}, \mathbf{G}]_{E}=\sum_{i, j=1}^{k_{E}} M_{E, i, j}\left(\mathbf{F}_{E}\right)^{e_{i}}\left(\mathbf{G}_{E}\right)^{e_{j}} \quad \forall \mathbf{F}, \mathbf{G} \in X^{d} \quad \forall E \in \mathcal{T}_{h} .
$$

Some minimal approximation properties for the scalar product (3.9) are required. We shall see in a while how a suitable matrix $M_{E}$ can be constructed. For the time being, we just assume that the scalar product (3.9) has the following property.

S1 (Stability of $[\cdot, \cdot]_{E}$ ). We assume that there exist two constants $s_{*}$ and $S^{*}$ independent of $h$ such that, for every $\mathbf{G} \in X^{d}$ and for every $E \in \mathcal{T}_{h}$, one has

$$
s_{*} \sum_{e \in \partial E}\left(G_{E}^{e}\right)^{2}|E| \leq[\mathbf{G}, \mathbf{G}]_{E} \leq S^{*} \sum_{e \in \partial E}\left(G_{E}^{e}\right)^{2}|E| .
$$


From (3.9) we can then easily construct the scalar product in $X^{d}$ by setting

$$
[\mathbf{F}, \mathbf{G}]_{X^{d}}=\sum_{E \in \mathcal{T}_{h}}[\mathbf{F}, \mathbf{G}]_{E} \quad \forall \mathbf{F}, \mathbf{G} \in X^{d}
$$

The third step of the MFD method is to derive an approximation to the divergence operator. The discrete divergence operator, $\mathcal{D} \mathcal{I} \mathcal{V}^{d}: X^{d} \rightarrow Q^{d}$, naturally arises from the Gauss divergence theorem as

$$
\left(\mathcal{D} \mathcal{I} \mathcal{V}^{d} \mathbf{F}\right)_{E} \stackrel{\text { def }}{=} \frac{1}{|E|} \sum_{e \in \partial E} F_{E}^{e}|e|
$$

We point out that our interpolation operators, in some sense, commute with the divergence operator. Indeed, for every vector field $\mathbf{G}$ smooth enough, we can use (3.12), (3.7), the Gauss divergence theorem, and (3.6) to obtain

$$
\left(\mathcal{D} \mathcal{I} \mathcal{V}^{d} \mathbf{G}^{I}\right)_{E}=\frac{1}{|E|} \sum_{e \in \partial E}\left(\mathbf{G}_{E}^{I}\right)^{e}|e|=\frac{1}{|E|} \int_{\partial E} \mathbf{G} \cdot \mathbf{n}_{E} \mathrm{~d} S=\frac{1}{|E|} \int_{E} \operatorname{div} \mathbf{G} \mathrm{d} V=(\operatorname{div} \mathbf{G})_{E}^{I}
$$

for every element $E$ in $\mathcal{T}_{h}$.

The fourth step of the MFD method is to define the discrete flux operator, $\mathcal{G}^{d}: Q^{d} \rightarrow X^{d}$, as the adjoint to the discrete divergence operator, $\mathcal{D} \mathcal{I} \mathcal{V}^{d}$, with respect to scalar products (3.8) and (3.11), i.e.

$$
\left[\mathbf{F}, \mathcal{G}^{d} \mathbf{p}\right]_{X^{d}}=\left[\mathbf{p}, \mathcal{D} \mathcal{I} \mathcal{V}^{d} \mathbf{F}\right]_{Q^{d}} \quad \forall \mathbf{p} \in Q^{d} \quad \forall \mathbf{F} \in X^{d}
$$

Using the discrete flux and divergence operators, the continuous problem (2.1), (2.2) is discretized as follows:

$$
\begin{aligned}
& \mathcal{D} \mathcal{I} \mathcal{V}^{d} \mathbf{F}_{d}=\mathbf{b} \\
& \mathbf{F}_{d}=\mathcal{G}^{d} \mathbf{p}_{d}
\end{aligned}
$$

where $\mathbf{b} \equiv \mathbf{b}^{I}$ is the vector of mean values of the source function $b$.

\section{Stability analysis}

In this section we analyze the stability of mimetic finite difference discretization (3.15)-(3.16) using the well-established methodology [5]. More precisely, we prove the coercivity condition (4.4) and the inf-sup condition (4.5).

Using the discrete Green formula (3.14), we rewrite equations (3.15), (3.16) in a form more suitable for the analysis:

$$
\begin{array}{ll}
{\left[\mathbf{F}_{d}, \mathbf{G}\right]_{X^{d}}-\left[\mathbf{p}_{d}, \mathcal{D} \mathcal{I} \mathcal{V}^{d} \mathbf{G}\right]_{Q^{d}}=0} & \forall \mathbf{G} \in X^{d} \\
{\left[\mathcal{D} \mathcal{I} \mathcal{V}^{d} \mathbf{F}_{d}, \mathbf{q}\right]_{Q^{d}}=[\mathbf{b}, \mathbf{q}]_{Q^{d}}} & \forall \mathbf{q} \in Q^{d}
\end{array}
$$

Let us introduce the following mesh norms on discrete spaces $X^{d}$ and $Q^{d}$ :

$$
\|\mathbf{p}\|\left\|_{Q^{d}}^{2}:=[\mathbf{p}, \mathbf{p}]_{Q^{d}}, \quad\right\| \mathbf{F} \|_{X^{d}}^{2}:=[\mathbf{F}, \mathbf{F}]_{X^{d}}
$$


and

$$
\left.\|\mathbf{F}\|\right|_{d i v} ^{2}:=\left.\|\mathbf{F}\|\right|_{X^{d}} ^{2}+\sum_{E \in \mathcal{T}_{h}} h_{E}^{2}\left\|\mathcal{D} \mathcal{I} \mathcal{V}^{d} \mathbf{F}\right\|_{L_{2}(E)}^{2}
$$

Let $V^{d}$ be the space of divergence-free discrete fluxes:

$$
V^{d}=\left\{\mathbf{F} \in X^{d}: \quad \mathcal{D} \mathcal{I} \mathcal{V}^{d} \mathbf{F}=0\right\}
$$

We begin the stability analysis by noticing that the scalar product (3.11) is continuous. It is also obvious that the scalar product satisfies the $V^{d}$-ellipticity condition:

$$
[\mathbf{F}, \mathbf{F}]_{X^{d}} \geq\|\mathbf{F}\|_{d i v}^{2} \quad \forall \mathbf{F} \in V^{d} .
$$

The analysis of the inf-sup condition is more involved. Following [5], for every $\mathbf{q} \in Q^{d}$, we have to find a vector $\mathbf{G} \in X^{d}$ such that

$$
\left[\mathcal{D} \mathcal{I} \mathcal{V}^{d} \mathbf{G}, \mathbf{q}\right]_{Q^{d}} \geq \beta_{*}\left|\|\mathbf{G}\|\left\|_{\text {div }}\right\|\right| \mathbf{q} \|_{Q^{d}}
$$

where $\beta_{*}$ is a constant independent of $\mathbf{q}, \mathbf{G}$, and $\mathcal{T}_{h}$. Let us denote by $q^{h} \in L_{2}(\Omega)$ the piecewise constant function on $\mathcal{T}_{h}$ with values given by the entries of the vector $\mathbf{q}$ (so that $\left(q^{h}\right)^{I} \equiv \mathbf{q}$ ). It is obvious that $\left\|q^{h}\right\|_{L_{2}(\Omega)}=\||\mathbf{q}|\|_{Q^{d}}$. Let us consider the homogeneous Dirichlet boundary value problem

$$
\Delta \psi=q^{h} \quad \text { in } \quad \Omega
$$

Since $\Omega$ has a Lipschitz-continuous boundary, there exist an $s>2$ and a constant $C_{\Omega}^{*}$ such that

$$
\|\psi\|_{W_{s}^{1}(\Omega)} \leq C_{\Omega}^{*}\left\|q^{h}\right\|_{L_{2}(\Omega)}
$$

Let $\mathbf{H}=\nabla \psi$, so that we have immediately

$$
\operatorname{div} \mathbf{H}=q^{h},
$$

and from (4.6)

$$
\|\mathbf{H}\|_{\left(L_{s}(\Omega)\right)^{3}}+\left(\sum_{E \in \mathcal{T}_{h}} h_{E}^{2}\|\operatorname{div} \mathbf{H}\|_{L_{2}(E)}^{2}\right)^{1 / 2} \leq\left(C_{\Omega}^{*}+h\right)\left\|q^{h}\right\|_{L_{2}(\Omega)} .
$$

We set now

$$
\mathbf{G}:=\mathbf{H}^{I} \equiv(\nabla \psi)^{I}
$$

where the interpolation operator is still the one defined in (3.7). Thanks to the commutative property (3.13) and to (4.7), we have

$$
\mathcal{D} \mathcal{I} \mathcal{V}^{d} \mathbf{G}=\left(q^{h}\right)^{I} \equiv \mathbf{q}
$$

Thus, inequality (4.5) is reduced to

$$
\left\|\left|\mathbf{q}\left\|_{Q^{d}} \geq \beta_{*} \mid\right\| \mathbf{G} \|_{\text {div }}\right.\right.
$$

At this point we need the technical lemma announced in the previous section. 
Lemma 4.1 Under the assumptions M1-M6 and S1, for every $s>2$ there exists a constant $\beta_{s}^{*}>0$ such that

$$
\left\|\mathbf{G}^{I}\right\|_{\text {div }} \leq \beta_{s}^{*}\left\{\|\mathbf{G}\|_{\left(L_{s}(\Omega)\right)^{3}}+\left(\sum_{E \in \mathcal{T}_{h}} h_{E}^{2}\|\operatorname{div} \mathbf{G}\|_{L_{2}(E)}^{2}\right)^{1 / 2}\right\}
$$

for every $\mathbf{G} \in\left(L_{s}(\Omega)\right)^{3}$ with $\operatorname{div} \mathbf{G} \in L_{2}(\Omega)$, and where $\mathbf{G}^{I}$ is defined in (3.7).

Proof. From (3.13) we immediately have

$$
\left\|\left|\mathcal{D} \mathcal{I} \mathcal{V}^{d} \mathbf{G}^{I}\right|\right\|_{Q^{d}}=\|\|(\operatorname{div} \mathbf{G})^{I}\|\|_{Q^{d}}=\|\operatorname{div} \mathbf{G}\|_{L_{2}(\Omega)}
$$

Therefore, in view of (4.3), it is sufficient to prove that there exists a constant $\widetilde{\beta_{s}^{*}}$ such that

$$
\left\|\mathbf{G}^{I}\right\|_{X^{d}} \leq \widetilde{\beta_{s}^{*}}\left\{\|\mathbf{G}\|_{\left(L_{s}(\Omega)\right)^{3}}+\left(\sum_{E \in \mathcal{T}_{h}} h_{E}^{2}\|\operatorname{div} \mathbf{G}\|_{L_{2}(E)}^{2}\right)^{1 / 2}\right\} .
$$

The desired result (4.12) follows from (4.14) with $\beta_{s}^{*}=\widetilde{\beta_{s}^{*}}+1$. In the following discussion we shall make a wide use of the conjugate exponent $t$, depending on $s$ through the usual formula

$$
\frac{1}{s}+\frac{1}{t}=1
$$

Assumption (3.10) implies clearly that

$$
\left[\mathbf{G}^{I}, \mathbf{G}^{I}\right]_{X^{d}} \leq S^{*} \sum_{E \in \mathcal{T}_{h}}|E| \sum_{e \in \partial E}\left(G_{E}^{e}\right)^{2}
$$

so that we have to estimate the $\left(G_{E}^{e}\right)$ 's in terms of $\mathbf{G}$, or, rather, in terms of the norm of $\mathbf{G}$ appearing in (4.12). Our basic instrument for that is called the lift property. The main difficulty, in the various cases, will be to prove that the lift property holds true.

$\mathbf{L P}$ (Lift Property). For every $t<2$ there exists a constant $\lambda^{*}=\lambda^{*}(t)$ such that: For every $E \in \mathcal{T}_{h}$ and for every $e \in \partial E$ there exists a function $\varphi_{E}^{e}$ from $E$ to $\mathbb{R}$ that verifies

$$
\varphi_{E}^{e}=1 \quad \text { on } \quad e, \quad \varphi_{E}^{e}=0 \quad \text { on } \quad \partial E \backslash e,
$$

and

$$
\left\|\varphi_{E}^{e}\right\|_{L_{2}(E)} \leq \lambda^{*} h_{E}^{3 / 2}, \quad\left\|\nabla \varphi_{E}^{e}\right\|_{\left(L_{t}(E)\right)^{3}} \leq \lambda^{*} h_{E}^{3 / t-1} .
$$

The lift property $\mathbf{L P}$ is proved in Appendix A.

Up to an approximation of $\mathbf{G}$ by smooth functions, and passage to the limit, we have, using (3.7), (4.17), and the Green formula:

$$
\begin{aligned}
G_{E}^{e}=\frac{1}{|e|} \int_{e} \mathbf{G} \cdot \mathbf{n}_{E} \mathrm{~d} S & =\frac{1}{|e|} \int_{\partial E} \varphi_{E}^{e} \mathbf{G} \cdot \mathbf{n}_{E} \mathrm{~d} S \\
& =\frac{1}{|e|} \int_{E} \mathbf{G} \cdot \nabla \varphi_{E}^{e} \mathrm{~d} V+\frac{1}{|e|} \int_{E} \varphi_{E}^{e} \operatorname{div} \mathbf{G} \mathrm{d} V .
\end{aligned}
$$


Using the Hölder inequality and (4.18) in (4.19), we have then

$$
\begin{aligned}
|e| G_{E}^{e} & \leq\|\mathbf{G}\|_{L_{s}(E)}\left\|\nabla \varphi_{E}^{e}\right\|_{L_{t}(E)}+\|\operatorname{div} \mathbf{G}\|_{L^{2}(E)}\left\|\varphi_{E}^{e}\right\|_{L^{2}(E)} \\
& \leq \lambda^{*}\left\{\left(h_{E}\right)^{3 / t-1}\|\mathbf{G}\|_{L_{s}(E)}+\left(h_{E}\right)^{3 / 2}\|\operatorname{div} \mathbf{G}\|_{L^{2}(E)}\right\} .
\end{aligned}
$$

Taking the squares and remembering that $(a+b)^{2} \leq 2\left(a^{2}+b^{2}\right)$, we have

$$
|e|^{2}\left(G_{E}^{e}\right)^{2} \leq 2\left(\lambda^{*}\right)^{2}\left\{\left(h_{E}\right)^{6 / t-2}\|\mathbf{G}\|_{L_{s}(E)}^{2}+\left(h_{E}\right)^{3}\|\operatorname{div} \mathbf{G}\|_{L^{2}(E)}^{2}\right\} .
$$

On the other hand, using conditions (2.4), we easily obtain

$$
|E| \leq h_{E}^{3}=h_{E}^{-1}\left(h_{E}^{2}\right)^{2} \leq h_{E}^{-1}\left(a^{*}\right)^{-2}|e|^{2} .
$$

We can now join (4.21) with (4.20) and deduce that

$$
\begin{aligned}
|E|\left(G_{E}^{e}\right)^{2} & \leq h_{E}^{-1}\left(a^{*}\right)^{-2}|e|^{2}\left(G_{E}^{e}\right)^{2} \\
& \leq \sigma^{*}\left\{\left(h_{E}\right)^{6 / t-3}\|\mathbf{G}\|_{L_{s}(E)}^{2}+\left(h_{E}\right)^{2}\|\operatorname{div} \mathbf{G}\|_{L^{2}(E)}^{2}\right\}
\end{aligned}
$$

where $\sigma^{*}=2\left(\lambda^{*}\right)^{2}\left(a^{*}\right)^{-2}$. Now we can sum (4.22) over all faces $e$ of $E$ and then over all elements $E$ of $\mathcal{T}_{h}$. We use (4.16) and Assumption M2 on the number of faces per element to get

$$
\begin{aligned}
&\|\| \mathbf{G}^{I}\|\|_{X^{d}}^{2} \leq N_{\ell} S^{*} \sigma^{*}\left\{\sum_{E \in \mathcal{T}_{h}}\left(h_{E}\right)^{6 / t-3}\|\mathbf{G}\|_{L_{s}(E)}^{2}+\sum_{E \in \mathcal{T}_{h}} h_{E}^{2}\|\operatorname{div} \mathbf{G}\|_{L_{2}(E)}^{2}\right\} \\
& \leq N_{\ell} S^{*} \sigma^{*}\left\{\left(\sum_{E \in \mathcal{T}_{h}}\left\{\left(h_{E}\right)^{6 / t-3}\right\}^{r}\right)^{1 / r}\left(\sum_{E \in \mathcal{T}_{h}}\|\mathbf{G}\|_{L_{s}(E)}^{s}\right)^{2 / s}\right. \\
&\left.+\sum_{E \in \mathcal{T}_{h}} h_{E}^{2}\|\operatorname{div} \mathbf{G}\|_{L_{2}(E)}^{2}\right\}
\end{aligned}
$$

where in the last step we applied the Hölder inequality with $r$, the conjugate exponent of $s / 2$,

$$
\frac{1}{r}+\frac{2}{s}=1
$$

A simple algebraic manipulation using (4.15) and (4.24) gives then

$$
\sum_{E \in \mathcal{T}_{h}}\left\{\left(h_{E}\right)^{6 / t-3}\right\}^{r}=\sum_{E \in \mathcal{T}_{h}} h_{E}^{3} \leq v_{*}^{-1}|\Omega|
$$

where we have also used (2.4) in the last step. Inserting (4.25) into (4.23), we finally get

$$
\left\|\mathbf{G}^{I}\right\|_{X^{d}} \leq \widetilde{\beta_{s}^{*}}\left\{\|\mathbf{G}\|_{\left(L_{s}(\Omega)\right)^{3}}+\left(\sum_{E \in \mathcal{T}_{h}} h_{E}^{2}\|\operatorname{div} \mathbf{G}\|_{L_{2}(E)}^{2}\right)^{1 / 2}\right\}
$$

where $\widetilde{\beta_{s}^{*}}$ depends only on $\lambda^{*}(t), v_{*}, a_{*}$ and $N_{\ell}$. This proves the assertion of the lemma.

Collecting (4.9) and (4.12), we get

$$
\|\mathbf{G}\|\left\|_{d i v}=\right\|\left|\mathbf{H}^{I}\right| \|_{d i v} \leq \beta_{s}^{*}\left\{\|\mathbf{H}\|_{\left(L_{s}(\Omega)\right)^{3}}+\left(\sum_{E \in \mathcal{T}_{h}} h_{E}^{2}\|\operatorname{div} \mathbf{H}\|_{L_{2}(E)}^{2}\right)^{1 / 2}\right\} .
$$

This, together with (4.8), implies (4.11) with $\beta_{*}=\left(\beta_{s}^{*}\left(C_{\Omega}^{*}+h\right)\right)^{-1}$. 


\section{Convergence analysis}

\subsection{Consistency assumption}

In order to prove error estimates, we need some assumptions on the scalar product (3.11), and more precisely on the relationships between the continuous scalar product (3.1) and its discrete counterpart (3.11). Our basic assumption will be the following one.

S2 (Consistency of $[,]_{E}$ ). For every element $E$, every linear function $q^{1}$ on $E$ and every $\mathbf{G} \in X^{d}$, we have

$$
\left[\left(\tilde{\mathbf{K}} \nabla q^{1}\right)^{I}, \mathbf{G}\right]_{E}=\int_{\partial E} q^{1} \mathbf{G}_{E} \cdot \mathbf{n}_{E} \mathrm{~d} S-\int_{E} q^{1}\left(\mathcal{D} \mathcal{I} \mathcal{V}^{d} \mathbf{G}\right)_{E} \mathrm{~d} V
$$

where $(\cdot)^{I}$ is the interpolation operator (3.7) and $\tilde{\mathbf{K}}$ is a constant tensor on $E$ such that

$$
\sup _{x \in E} \sup _{i, j}\left|\{\mathbf{K}(\mathbf{x})\}_{i, j}-\{\tilde{\mathbf{K}}\}_{i, j}\right| \leq C_{K}^{*} h_{E}
$$

where $C_{K}^{*}$ is a constant independent of $E$.

Note that $\tilde{\mathbf{K}}$ may be any reasonable piecewise constant approximation of $\mathbf{K}$. In practice, we use either the value of $\mathbf{K}$ at the polyhedron mass center or its mean value.

Condition (5.1) is rather new and requires some comments. First, we point out that we shall need it to hold only for vectors $\mathbf{G} \in V^{d}$. For divergence-free vectors (5.1) reads

$$
\left[\left(\tilde{\mathbf{K}} \nabla q^{1}\right)^{I}, \mathbf{G}\right]_{E}=\int_{\partial E} q^{1} \mathbf{G}_{E} \cdot \mathbf{n}_{E} \mathrm{~d} S,
$$

showing the remarkable property of using only boundary integrals. However, as $\mathcal{D I} \mathcal{V}^{d} \mathbf{G}$ is constant in each $E$ and $q^{1}$ is supposed to be linear, the volume integral appearing in (5.1) is not difficult to compute. Taking $\mathbf{G}=\left(\tilde{\mathbf{K}} \nabla \tilde{q}^{1}\right)^{I}$ (with $\tilde{q}^{1}$ another polynomial of degree $\leq 1$ ) in (5.3), we conclude that assumption $\mathbf{S} 2$ implies that the scalar product (3.11) is exact for constant velocities and constant tensors.

In the context of the local mimetic finite difference method [12], condition (5.1) means that the discrete gradient operator is exact for linear functions. This property has been used in [17] to build a one-parameter family of symmetric positive definite matrices $M_{E}$ for a triangle. As a particular case, the family includes the mass matrix appearing in the finite element discretizations with the Raviart-Thomas finite elements.

What is still remarkable in (5.1) is that it does not require the construction of a lifting operator from the values $G_{E}^{e}$ on $\partial E$ to the interior of $E$. It is not difficult to show, however, that if we have any reasonable lifting operator $R_{E}$, then the choice

$$
[\mathbf{F}, \mathbf{G}]_{E}:=\int_{E} \tilde{\mathbf{K}}^{-1} R_{E}\left(\mathbf{F}_{E}\right) \cdot R_{E}\left(\mathbf{G}_{E}\right) \mathrm{d} V
$$

will automatically satisfy (5.1) as well as (3.10). We have indeed the following proposition. 
Theorem 5.1 Assume that for every element $E \in \mathcal{T}_{h}$ we have a lifting operator $R_{E}$ acting on $X_{E}^{d}$ (the restriction of $X^{d}$ to $E$ ) and with values in $\left(L_{2}(E)\right)^{3}$ such that

$$
\begin{aligned}
R_{E}\left(\mathbf{G}_{E}\right) \cdot \mathbf{n}_{E} & \equiv \mathbf{G}_{E} \cdot \mathbf{n}_{E} & \text { on } \partial E \\
\operatorname{div} R_{E}\left(\mathbf{G}_{E}\right) & \equiv\left(\mathcal{D} \mathcal{I} \mathcal{V}^{d} \mathbf{G}\right)_{E} & \text { in } E
\end{aligned}
$$

for all $\mathbf{G} \in X^{d}$, and

$$
R_{E}\left(\mathbf{G}_{E}^{I}\right)=\mathbf{G}
$$

for all $\mathbf{G}$ constant on $E$. Then the choices

$$
\{\tilde{\mathbf{K}}\}_{i, j}:=\frac{1}{|E|} \int_{E}\{\mathbf{K}\}_{i, j} \mathrm{~d} V
$$

and

$$
[\mathbf{F}, \mathbf{G}]_{E}:=\int_{E} \tilde{\mathbf{K}}^{-1} R_{E}\left(\mathbf{F}_{E}\right) \cdot R_{E}\left(\mathbf{G}_{E}\right) \mathrm{d} V
$$

will automatically satisfy (5.2) and (5.1). If moreover there exist two constants $c_{R}^{*}$ and $C_{R}^{*}$, independent of E, such that

$$
c_{R}^{*}\left(|E| \sum_{e \in \partial E}\left(G_{E}^{e}\right)^{2}\right)^{1 / 2} \leq\left\|R_{E}(\mathbf{G})\right\|_{\left(L_{2}(E)\right)^{3}} \leq C_{R}^{*}\left(|E| \sum_{e \in \partial E}\left(G_{E}^{e}\right)^{2}\right)^{1 / 2}
$$

for all $\mathbf{G} \in X^{d}$, then (3.10) will also hold with constants $s_{*}$ and $S^{*}$ depending only on $c_{R}^{*}, C_{R}^{*}$ and on the constants $\kappa_{*}, \kappa^{*}$ from (2.3).

Proof. The validity of (5.2) is immediate. The validity of (5.1) is also easily checked:

$$
\begin{array}{rlrl}
{\left[\left(\tilde{\mathbf{K}} \nabla q^{1}\right)^{I}, \mathbf{G}\right]_{E}} & =\int_{E} \tilde{\mathbf{K}}^{-1} R_{E}\left(\left(\tilde{\mathbf{K}} \nabla q^{1}\right)_{E}^{I}\right) \cdot R_{E}\left(\mathbf{G}_{E}\right) \mathrm{d} V & & \text { (use (5.5) and } \nabla q^{1}=\text { const) } \\
& =\int_{E} \tilde{\mathbf{K}}^{-1} \tilde{\mathbf{K}} \nabla q^{1} \cdot R_{E}\left(\mathbf{G}_{E}\right) \mathrm{d} V & & \text { (use } \left.\tilde{\mathbf{K}}^{-1} \tilde{\mathbf{K}}=I d\right) \\
& =\int_{E} \nabla q^{1} \cdot R_{E}\left(\mathbf{G}_{E}\right) \mathrm{d} V & & \text { (integrate by parts) } \\
& =\int_{\partial E} q^{1} R_{E}\left(\mathbf{G}_{E}\right) \cdot \mathbf{n}_{E} \mathrm{~d} S-\int_{E} q^{1} \operatorname{div} R_{E}\left(\mathbf{G}_{E}\right) \mathrm{d} V & & \text { (use (5.4)) } \\
& =\int_{\partial E} q^{1} \mathbf{G}_{E} \cdot \mathbf{n}_{E} \mathrm{~d} S-\int_{E} q^{1}\left(\mathcal{D} \mathcal{I} \mathcal{V}^{d} \mathbf{G}\right)_{E} \mathrm{~d} V . &
\end{array}
$$

Finally, (3.10) follows immediately from (5.7), (2.3) and (5.8) after noting that (2.3) is equivalent to

$$
\left(\kappa^{*}\right)^{-1}\|\mathbf{v}\|^{2} \leq \mathbf{v}^{T} \mathbf{K}^{-1}(\mathbf{x}) \mathbf{v} \leq\left(\kappa_{*}\right)^{-1}\|\mathbf{v}\| \quad \forall \mathbf{v} \in \mathbb{R}^{3} \quad \forall \mathbf{x} \in \Omega .
$$

This ends the proof of the theorem.

A possible way of getting (5.1) is therefore to construct a lifting operator $R_{E}$ satisfying (5.4), (5.5), and (5.8), and then define $M_{E}$ following (5.7). For instance, the way followed in [15] for polygonal domains can be interpreted as the construction of a lifting operator satisfying (5.4) and (5.5). 
In general, we may consider assumption (5.1) as a system of linear equations where the unknowns are the coefficients of $M_{E}$, and use it, in each element $E$, to construct the matrix $M_{E}$. Since the matrix $M_{E}$ should be symmetric and positive definite, this is a problem with nonlinear constraints. An analytical solution has been found only for triangular elements [17].

Let us see this in more detail. We consider an element $E$ having $k_{E}$ faces. Equation (5.1) should then hold for $k_{E}$ different possible choices of $\mathbf{G}_{E}$ and three possible choices of $q^{1}$ corresponding to $q^{1}=x, q^{1}=y$ and $q^{1}=z$. Note that for $q^{1}=1$ the equation (5.1) is automatically satisfied as it reduces to our definition of the operator $\mathcal{D} \mathcal{I} \mathcal{V}^{d}$. We have therefore $3 k_{E}$ equations. Since $\tilde{\mathbf{K}}$, and hence $M_{E}$, are symmetric, the number of unknown coefficients of $M_{E}$ is $\left(k_{E}+1\right)\left(k_{E}+2\right) / 2$, that is bigger than $3 k_{E}$ as soon as $k_{E} \geq 3$. The system will always be compatible, since we could always define a lifting $R_{E}$ first by solving, for each $\mathbf{G}_{E}$, the Neumann problem,

$$
\begin{array}{ll}
\Delta \chi=\mathcal{D} \mathcal{I} \mathcal{V}^{d} \mathbf{G}_{E} & \text { in } \quad E \\
\partial \chi / \partial \mathbf{n}_{E}=\mathbf{G}_{E} \cdot \mathbf{n}_{E} & \text { on } \quad \partial E,
\end{array}
$$

then by taking $R_{E}\left(\mathbf{G}_{E}\right):=\nabla \chi$, and finally by defining $M_{E}$ through (5.7). This will be totally impractical, but shows that at least a solution $M_{E}$ of (5.1), symmetric and positive definite, exists (although, in general, the solution will not be unique.)

Particular structures could be imposed to $M_{E}$ in order to reduce the number of unknowns. For instance, we can require that each face interacts only with two neighboring faces, reducing the number of unknowns to $3 k_{E}$, that equals the number of equations and makes the linear system much easier to solve on the computer.

An advantage of this approach is that it can rather easily be extended to faces that are not flat. This is a case in which the construction of an explicit lifting operator might prove to be very difficult. We shall address this problem in the future publications.

\subsection{Error estimate for the vector variable}

Using Assumption $\mathbf{S 2}$, we are going to prove error estimates for our discretization. Let $(p, \mathbf{F})$ be the exact solution of (2.1) and (2.2), let $\left(\mathbf{p}_{d}, \mathbf{F}_{d}\right)$ be the discrete solution (see (3.15) and (3.16)), and let $\mathbf{p}^{I}$ and $\mathbf{F}^{I}$ be the interpolants of the exact solution. Finally, for every element $E$, we denote by $p_{E}^{1}$ a suitable polynomial of degree $\leq 1$ that approximates $p$, and that will be decided later on. We notice first that from (2.1), (3.13) and (3.15), we easily have:

$$
\mathcal{D I} \mathcal{V}^{d}\left(\mathbf{F}^{I}-\mathbf{F}_{d}\right)=\mathbf{b}-\mathbf{b}=0
$$

Using (2.2) and (3.16), then (3.14), and finally (5.10), we get

$$
\begin{aligned}
{\left[\mathbf{F}^{I}-\mathbf{F}_{d}, \mathbf{F}^{I}-\mathbf{F}_{d}\right]_{X^{d}} } & =\left[(-\mathbf{K} \nabla p)^{I}, \mathbf{F}^{I}-\mathbf{F}_{d}\right]_{X^{d}}-\left[\mathcal{G}^{d} \mathbf{p}_{d}, \mathbf{F}^{I}-\mathbf{F}_{d}\right]_{X^{d}} \\
& =\left[(-\mathbf{K} \nabla p)^{I}, \mathbf{F}^{I}-\mathbf{F}_{d}\right]_{X^{d}}-\left[\mathbf{p}_{d}, \mathcal{D} \mathcal{I} \mathcal{V}^{d}\left(\mathbf{F}^{I}-\mathbf{F}_{d}\right)\right]_{Q^{d}} \\
& =\left[(-\mathbf{K} \nabla p)^{I}, \mathbf{F}^{I}-\mathbf{F}_{d}\right]_{X^{d}}
\end{aligned}
$$


Then, adding and subtracting terms we have

$$
\begin{aligned}
\left\|\mathbf{F}^{I}-\mathbf{F}_{d}\right\| \|_{X^{d}}^{2} & =\left[(-\mathbf{K} \nabla p)^{I}+\left(\mathbf{K} \nabla p^{1}\right)^{I}, \mathbf{F}^{I}-\mathbf{F}_{d}\right]_{X^{d}}+\left[\left(-\mathbf{K} \nabla p^{1}\right)^{I}, \mathbf{F}^{I}-\mathbf{F}_{d}\right]_{X^{d}} \\
& =\mathbf{I}_{1}+\left[\left(-\mathbf{K} \nabla p^{1}+\tilde{\mathbf{K}} \nabla p^{1}\right)^{I}, \mathbf{F}^{I}-\mathbf{F}_{d}\right]_{X^{d}}+\left[\left(-\tilde{\mathbf{K}} \nabla p^{1}\right)^{I}, \mathbf{F}^{I}-\mathbf{F}_{d}\right]_{X^{d}} \\
& =\mathbf{I}_{1}+\mathbf{I}_{2}+\left[\left(-\tilde{\mathbf{K}} \nabla p^{1}\right)^{I}, \mathbf{F}^{I}-\mathbf{F}_{d}\right]_{X^{d}} \\
& =\mathbf{I}_{1}+\mathbf{I}_{2}+\mathbf{I}_{3} .
\end{aligned}
$$

Using (5.1) and (5.10), the third term reads:

$$
\begin{aligned}
\mathbf{I}_{3} & =\sum_{E \in \mathcal{T}_{h}}\left\{\int_{\partial E} p_{E}^{1}\left(\mathbf{F}^{I}-\mathbf{F}_{d}\right)_{E} \cdot \mathbf{n}_{E} \mathrm{~d} S-\int_{E} p_{E}^{1}\left(\mathcal{D} \mathcal{I} \mathcal{V}^{d}\left(\mathbf{F}^{I}-\mathbf{F}_{d}\right)\right)_{E} \mathrm{~d} V\right\} \\
& =\sum_{E \in \mathcal{T}_{h}} \int_{\partial E} p_{E}^{1}\left(\mathbf{F}^{I}-\mathbf{F}_{d}\right)_{E} \cdot \mathbf{n}_{E} \mathrm{~d} S
\end{aligned}
$$

We are therefore left with the problem of estimating $\mathbf{I}_{1}, \mathbf{I}_{2}$, and $\mathbf{I}_{3}$. A first estimate of $\mathbf{I}_{2}$ is trivial. From (5.2) we immediately have

$$
\mathbf{I}_{2} \equiv\left[\left(-\mathbf{K} \nabla p^{1}+\tilde{\mathbf{K}} \nabla p^{1}\right)^{I}, \mathbf{F}^{I}-\mathbf{F}_{d}\right]_{X^{d}} \leq C_{K}^{*} h\left\|\left|\left(\nabla p^{1}\right)^{I}\right|||_{X^{d}}\right\| \mid \mathbf{F}^{I}-\mathbf{F}_{d} \|_{X^{d}}
$$

where $p^{1}$ still has to be defined.

Let us recall some known properties on the approximation errors. For the sake of simplicity, we assume that our solution $p$ is in $H^{2}(\Omega)$. Note that with a little additional effort we could use a weaker regularity, and get a lower order of convergence.

We first recall that, under Assumption M6 (Star-shaped elements), it is possible to find a constant $C_{a p p}^{*}$, depending only on $\tau_{*}$, such that for every element $E$ and for every $p \in H^{2}(E)$ there exist a constant $p_{E}^{0}$ and a polynomial $p_{E}^{1}$ of degree $\leq 1$ such that

$$
\begin{gathered}
\left\|p-p_{E}^{0}\right\|_{L_{2}(E)} \leq C_{a p p}^{*} h_{E}\|p\|_{H^{1}(E)}, \\
\left\|p-p_{E}^{1}\right\|_{L_{2}(E)} \leq C_{a p p}^{*} h_{E}^{2}\|p\|_{H^{2}(E)}, \quad\left\|p-p_{E}^{1}\right\|_{H^{1}(E)} \leq C_{a p p}^{*} h_{E}\|p\|_{H^{2}(E)}
\end{gathered}
$$

(see [4, Lemma 4.3.8]). Concerning the error on faces, we can use a result due to Agmon and made popular in the numerical analysis community by D.N. Arnold [1]. Applied to our case, it says that there exists a constant $C_{a g m}^{*}$, depending only on the constant $\gamma_{*}$ of Assumption M4, such that for every pyramid $P_{E}^{e}$ (as described in Assumption M5), and for every function $\chi \in H^{1}\left(P_{E}^{e}\right)$, we have

$$
\|\chi\|_{L_{2}(e)}^{2} \leq C_{a g m}^{*}\left(h_{E}^{-1}\|\chi\|_{L_{2}\left(P_{E}^{e}\right)}^{2}+h_{E}\|\chi\|_{H^{1}\left(P_{E}^{e}\right)}^{2}\right) .
$$

It is then immediate to derive from (5.17) that

$$
\|\nabla \chi\|_{L_{2}(e)}^{2} \leq C_{a g m}^{*}\left(h_{E}^{-1}\|\chi\|_{H^{1}\left(P_{E}^{e}\right)}^{2}+h_{E}\|\chi\|_{H^{2}\left(P_{E}^{e}\right)}^{2}\right)
$$

for every $\chi \in H^{2}(E)$. Applying this to the difference $p-p_{E}^{1}$, and using (5.16), we get:

$$
\left\|p-p_{E}^{1}\right\|_{L_{2}(e)}^{2}+h_{E}^{2}\left\|\nabla\left(p-p_{E}^{1}\right)\right\|_{L_{2}(e)}^{2} \leq C_{f a c e}^{*} h_{E}^{3}\|p\|_{H^{2}(E)}^{2}
$$


where $C_{\text {face }}^{*}$ depends only on $\tau_{*}$ and $\gamma_{*}$.

Now, we can finish the estimate of $\mathbf{I}_{2}$. Note that $\nabla p^{1}$ is a constant vector. Then, (5.16) and the triangle inequality give:

$$
\left\|\left(\nabla p_{E}^{1}\right)^{I} \mid\right\|_{X^{d}}=\left\|\nabla p_{E}^{1}\right\|_{L_{2}(E)} \leq\|\nabla p\|_{L_{2}(E)}+\left\|\nabla\left(p-p_{E}^{1}\right)\right\|_{L_{2}(E)} \leq\left(1+h_{E} C_{a p p}^{*}\right)\|p\|_{H^{2}(E)} .
$$

Thus, we obtain immediately from (5.14) that

$$
\mathbf{I}_{2} \leq C_{I_{2}}^{*} h\|p\|_{H^{2}(\Omega)}\left\|\mid \mathbf{F}^{I}-\mathbf{F}_{d}\right\|_{X^{d}}
$$

where $C_{I_{2}}^{*}$ equals to $\left(1+h_{E} C_{a p p}^{*}\right) C_{K}^{*}$ with $C_{K}^{*}$ given in (5.2).

The estimate of $I_{1}$ is obtained in the following lemma.

Lemma 5.1 Let $p \in H^{2}(\Omega)$ and let, in each $E \in \mathcal{T}_{h}$, $p^{1}$ be such that (5.16) holds. Let $(\cdot)^{I}$ be the interpolation operator defined in (3.7), and let finally $\mathbf{G} \in X^{d}$. Then

$$
\left[(-\mathbf{K} \nabla p)^{I}+\left(\mathbf{K} \nabla p^{1}\right)^{I}, \mathbf{G}\right]_{X^{d}} \leq C_{I_{1}}^{*} h\|p\|_{H^{2}(\Omega)} \mid\|\mathbf{G}\|_{X^{d}}
$$

where the constant $C_{I_{1}}^{*}$ is independent of $p, \mathbf{G}$ and $h$.

Proof. The proof follows immediately from (3.10), the definition of the interpolation operator (3.7), the Cauchy-Schwartz inequality, and the approximation results quoted above. Indeed, we have

$$
\begin{aligned}
\|(-\mathbf{K} \nabla p)^{I}+\left(\mathbf{K} \nabla p^{1}\right)^{I}||_{X^{d}}^{2} & \leq S^{*} \sum_{E \in \mathcal{T}_{h}} \sum_{e \in \partial E}\left(\left((-\mathbf{K} \nabla p)^{I}+\left(\mathbf{K} \nabla p^{1}\right)^{I}\right)_{E}^{e}\right)^{2}|E| \\
& \leq S^{*} \sum_{E \in \mathcal{T}_{h}} \sum_{e \in \partial E}\left(\frac{1}{|e|} \int_{e} \mathbf{K} \nabla\left(p-p_{E}^{1}\right) \cdot \mathbf{n}_{E} \mathrm{~d} S\right)^{2}|E| \\
& \leq S^{*} \sum_{E \in \mathcal{T}_{h}} \sum_{e \in \partial E} \frac{1}{|e|}\left\|\mathbf{K} \nabla\left(p-p_{E}^{1}\right)\right\|_{L_{2}(e)}^{2}|E| \\
& \leq C_{I_{1}}^{*} h^{2}\|p\|_{H^{2}(\Omega)}^{2}
\end{aligned}
$$

where $C_{I_{1}}^{*}$ depends only on $a_{*}$ given in (2.4), $S^{*}$ given in (3.10), $\kappa^{*}$ given in (2.3), and $C_{f a c e}^{*}$ obtained in (5.19).

The following Lemma gives an estimate for $\mathbf{I}_{3}$.

Lemma 5.2 Let $p \in H^{2}(\Omega)$ and let, in each $E \in \mathcal{T}_{h}$, $p^{1}$ be such that (5.16) holds. Moreover let $\mathrm{G} \in X^{d}$. Then

$$
\sum_{E \in \mathcal{T}_{h}} \int_{\partial E} p^{1} \mathbf{G}_{E} \cdot \mathbf{n}_{E} \mathrm{~d} S \leq C_{I_{3}}^{*} h\|p\|_{H^{2}(\Omega)}\|\mathbf{G}\| \|_{X^{d}}
$$

where the constant $C_{I_{3}}^{*}$ is independent of $p, \mathbf{G}$ and $h$. 
Proof. The first (crucial) step of the proof uses the continuity of $p$ and the fact that $\mathbf{G}_{E} \cdot \mathbf{n}_{E}$ takes opposite values for the two elements sharing each internal face; then the result follows with usual instruments, like the Cauchy-Schwartz inequality and the approximation results (5.16):

$$
\begin{aligned}
\sum_{E \in \mathcal{T}_{h}} \int_{\partial E} p_{E}^{1} \mathbf{G}_{E} \cdot \mathbf{n}_{E} \mathrm{~d} S & =\sum_{E \in \mathcal{T}_{h}} \int_{\partial E}\left(p_{E}^{1}-p\right) \mathbf{G}_{E} \cdot \mathbf{n}_{E} \mathrm{~d} S \\
& \leq \sum_{E \in \mathcal{T}_{h}} \sum_{e \in \partial E}\left\|p-p_{E}^{1}\right\|_{L_{2}(e)}\left\|G_{E}^{e}\right\|_{L_{2}(e)} \\
& =\sum_{E \in \mathcal{T}_{h}} \sum_{e \in \partial E}\left\|p-p_{E}^{1}\right\|_{L_{2}(e)}\left|G_{E}^{e}\right||e|^{1 / 2} \\
& \leq v_{*}^{-1 / 2}\left(C_{f a c e}^{*}\right)^{1 / 2} \sum_{E \in \mathcal{T}_{h}} h_{E}\|p\|_{H_{2}(E)} \sum_{e \in \partial E}\left|G_{E}^{e}\right||E|^{1 / 2} \\
& \leq C_{I_{3}}^{*} h\|p\|_{H^{2}(\Omega)}\|\mid \mathbf{G}\|_{X^{d}} .
\end{aligned}
$$

where $C_{I_{3}}^{*}=\left(v_{*}^{-1} s_{*}^{-1} C_{f a c e}^{*}\right)^{1 / 2} N_{\ell}$. This proves the assertion of the lemma.

Combining (5.12) with (5.20), (5.21) and (5.22), we finally get the main convergence result.

Theorem 5.2 Under assumptions P1, M1-M6 and S1-S2, let $(p, \mathbf{F})$ be the solution of (2.1)(2.2), and let $\left(p_{d}, \mathbf{F}_{d}\right)$ be the discrete solution, given by (3.15)-(3.16). Let moreover $\mathbf{F}^{I}$ be the interpolant of $\mathbf{F}$, introduced in (3.7). Then we have

$$
\left\|\mid \mathbf{F}^{I}-\mathbf{F}_{d}\right\|_{X^{d}} \leq C^{*} h\|p\|_{H^{2}(\Omega)},
$$

where $C^{*}$ depends only upon the various constants appearing in Assumptions P1, M1-M6 and S1-S2.

\subsection{Error estimates for the scalar variable}

For the estimate on the scalar variable $\mathbf{p}_{d}$, we shall go back to the inf-sup condition (4.5). For the sake of simplicity, we assume that $\Omega$ is convex. Let $\psi$ be the solution of

$$
\begin{aligned}
-\operatorname{div}(\mathbf{K} \nabla \psi) & =\mathbf{p}^{I}-\mathbf{p}_{d} & & \text { in } \Omega \\
\psi & =0 & & \text { on } \partial \Omega
\end{aligned}
$$

where, for simplicity, we identified $\mathbf{p}_{d}-\mathbf{p}^{I}$ with the corresponding piecewise constant function. The convexity of $\Omega$ implies that there exists a constant $C_{\Omega}^{*}$, depending only on $\Omega$, such that

$$
\|\psi\|_{H^{2}(\Omega)} \leq C_{\Omega}^{*}\left\|\left|\mathbf{p}_{d}-\mathbf{p}^{I}\right|\right\|_{Q^{d}}
$$

We set now

$$
\mathbf{H}=\mathbf{K} \nabla \psi
$$

and define $\mathbf{G} \in X^{d}$ as $\mathbf{G}=\mathbf{H}^{I}$, so that

$$
\mathcal{D I} \mathcal{V}^{d} \mathbf{G}=\mathbf{p}_{d}-\mathbf{p}^{I}
$$


Finally, we denote by $\psi^{1}$ a piecewise linear approximation of $\psi$ that satisfy (5.16) for each $E \in \mathcal{T}_{h}$. Using (5.26), then (4.1), then (3.6) and (3.13), then integrating by parts, and finally integrating once more by parts and using (2.1) and (2.2) we get

$$
\begin{aligned}
\left\|\mathbf{p}_{d}-\mathbf{p}^{I}\right\|_{Q^{d}}^{2} & =\left[\mathcal{D} \mathcal{I} \mathcal{V}^{d} \mathbf{G}, \mathbf{p}_{d}-\mathbf{p}^{I}\right]_{Q^{d}} \\
& =\left[\mathbf{F}_{d}, \mathbf{G}\right]_{X^{d}}-\left[\mathcal{D} \mathcal{I} \mathcal{V}^{d} \mathbf{G}, \mathbf{p}^{I}\right]_{Q^{d}}=\left[\mathbf{F}_{d}, \mathbf{G}\right]_{X^{d}}-\int_{\Omega} p \operatorname{div}(\mathbf{K} \nabla \psi) \mathrm{d} V \\
& =\left[\mathbf{F}_{d}, \mathbf{G}\right]_{X^{d}}+\int_{\Omega} \mathbf{K} \nabla p \cdot \nabla \psi \mathrm{d} V \\
& =\left[\mathbf{F}_{d}, \mathbf{G}\right]_{X^{d}}+\int_{\Omega} b \psi \mathrm{d} V .
\end{aligned}
$$

Now, using the definition of $\mathbf{G}$ and adding and subtracting terms, we have

$$
\begin{aligned}
\left\|\mathbf{p}_{d}-\mathbf{p}^{I}\right\|_{Q^{d}}^{2} & =\left[\mathbf{F}_{d},(\mathbf{K} \nabla \psi)^{I}-\left(\mathbf{K} \nabla \psi^{1}\right)^{I}\right]_{X^{d}}+\left[\mathbf{F}_{d},\left(\mathbf{K} \nabla \psi^{1}\right)^{I}\right]_{X^{d}}+\int_{\Omega} b \psi \mathrm{d} V \\
& =J_{1}+\left[\mathbf{F}_{d},\left((\mathbf{K}-\tilde{\mathbf{K}}) \nabla \psi^{1}\right)^{I}\right]_{X^{d}}+\left[\mathbf{F}_{d},\left(\tilde{\mathbf{K}} \nabla \psi^{1}\right)^{I}\right]_{X^{d}}+\int_{\Omega} b \psi \mathrm{d} V \\
& =J_{1}+J_{2}+\left[\mathbf{F}_{d},\left(\tilde{\mathbf{K}} \nabla \psi^{1}\right)^{I}\right]_{X^{d}}+\int_{\Omega} b \psi \mathrm{d} V
\end{aligned}
$$

Using (5.21), the term $J_{1}$ can be easily bounded by

$$
J_{1} \equiv\left[\mathbf{F}_{d},(\mathbf{K} \nabla \psi)^{I}-\left(\mathbf{K} \nabla \psi^{1}\right)^{I}\right]_{X^{d}} \leq C_{I_{1}}^{*} h\left\|\mid \mathbf{F}_{d}\right\|_{X^{d}}\|\psi\|_{H^{2}(\Omega)} .
$$

The term $J_{2}$ is bounded as in (5.14), (5.20) by

$$
J_{2} \equiv\left[\mathbf{F}_{d},\left((\mathbf{K}-\tilde{\mathbf{K}}) \nabla \psi^{1}\right)^{I}\right]_{X^{d}} \leq C_{I_{2}}^{*} h\left\|\mid \mathbf{F}_{d}\right\|_{X^{d}}\|\psi\|_{H^{2}(\Omega)} .
$$

For the third term in the last line of (5.27) we can use (5.1) to obtain

$$
\left[\mathbf{F}_{d},\left(\mathbf{K} \nabla \psi^{1}\right)^{I}\right]_{X^{d}}=\sum_{E \in \mathcal{T}_{h}} \int_{\partial E} \psi^{1}\left(\mathbf{F}_{d}\right)_{E} \cdot \mathbf{n}_{E} \mathrm{~d} S-\int_{\Omega} \mathbf{b} \psi^{1} \mathrm{~d} V
$$

With the help of (5.22), we get then

$$
\left|\left[\mathbf{F}_{d},\left(\tilde{\mathbf{K}} \nabla \psi^{1}\right)^{I}\right]_{X^{d}}+\int_{\Omega} b \psi \mathrm{d} V\right| \leq C_{I_{3}}^{*} h||\left|\mathbf{F}_{d}\right|\left\|_{X^{d}}\right\| \psi \|_{H^{2}(\Omega)}+\left|\int_{\Omega}\left(b \psi-\mathbf{b} \psi^{1}\right) \mathrm{d} V\right|
$$

where the last term is easily bounded by $2 C_{a p p}^{*} h\|b\|_{H^{1}(\Omega)}\|\psi\|_{H^{1}(\Omega)}$. Collecting the above inequalities (5.27) - (5.31), we easily obtain

$$
\left\|\left|\mathbf{p}_{d}-\mathbf{p}^{I}\right|\right\|_{Q^{d}}^{2} \leq C^{*} h\left\{\left\|\mid \mathbf{F}_{d}\right\|\left\|_{X^{d}}+\right\| b \|_{H^{1}(\Omega)}\right\}\|\psi\|_{H^{2}(\Omega)}
$$

that combined with the estimates (5.24), Theorem 5.2 and Lemma 4.1 gives the proof of the second convergence result. 
Theorem 5.3 Under assumptions of Theorem 5.2, plus the convexity of $\Omega$, we have

$$
\left.\left\|\mathbf{p}_{d}-\mathbf{p}^{I}\right\|\right|_{Q^{d}} \leq C^{*} h\left(\|p\|_{H^{2}(\Omega)}+\|b\|_{H^{1}(\Omega)}\right)
$$

where the constant $C^{*}$ depends only on the constants appearing in Assumptions P1, M1-M6 and S1-S2, on $C_{\Omega}^{*}$ appearing in (5.24) and on $\beta_{s}^{*}$ appearing in (4.12).

It is interesting to note that, assuming that in each element $E$ we had a suitable lifting $R_{E}$, a better estimate for the scalar variable could be obtained. We have indeed the following theorem

Theorem 5.4 Together with the assumptions of Theorem 5.3, assume moreover that for each element $E$ we have a lifting operator $R_{E}$ with the properties (5.4), (5.5) and (5.8) such that

$$
\left\|R_{E}\left(\mathbf{G}^{I}\right)-\mathbf{G}\right\|_{L_{2}(E)} \leq C_{R a}^{*} h_{E}\|\mathbf{G}\|_{\left(H^{1}(E)\right)^{3}} \quad \forall \mathbf{G} \in\left(H^{1}(E)\right)^{3} \quad \forall E \in \mathcal{T}_{h}
$$

where $C_{R a}^{*}$ is a constant independent of $\mathbf{G}$ and $h_{E}$. Then, we have

$$
\left\|\left|\mathbf{p}_{d}-\mathbf{p}^{I}\right|\right\|_{Q^{d}} \leq C^{*} h^{2}\left(\|p\|_{H^{2}(\Omega)}+\|b\|_{H^{1}(\Omega)}\right)
$$

where the constant $C^{*}$ depends only on the constants appearing in Assumptions P1, M1-M6 and $\mathbf{S 1 - S 2}$, on $C_{\Omega}^{*}$ appearing in (5.24), on $\beta_{s}^{*}$ appearing in (4.12), and on $C_{R a}^{*}$ appearing in (5.34).

Proof. Let $R(\mathbf{G})$ be such that $\left.R(\mathbf{G})\right|_{E}=R_{E}\left(\mathbf{G}_{E}\right)$. Following essentially [10] and using (5.26), then (4.1), (3.6) and (3.13) (as in the previous proof) with (5.4), then integrating by parts and finally using (2.2) and (5.7), we get

$$
\begin{aligned}
\left\|\mathbf{p}_{d}-\mathbf{p}^{I}\right\|_{Q^{d}}^{2} & =\left[\mathcal{D} \mathcal{I} \mathcal{V}^{d} \mathbf{G}, \mathbf{p}_{d}-\mathbf{p}^{I}\right]_{Q^{d}} \\
& =\left[\mathbf{F}_{d}, \mathbf{G}\right]_{X^{d}}-\int_{\Omega} p \operatorname{div} R(\mathbf{G}) \mathrm{d} V \\
& =\left[\mathbf{F}_{d}, \mathbf{G}\right]_{X^{d}}+\int_{\Omega} \nabla p \cdot R(\mathbf{G}) \mathrm{d} V=\left[\mathbf{F}_{d}, \mathbf{G}\right]_{X^{d}}+\int_{\Omega} \mathbf{K}^{-1} \mathbf{K} \nabla p \cdot R(\mathbf{G}) \mathrm{d} V \\
& =\int_{\Omega} \mathbf{K}^{-1}\left(R\left(\mathbf{F}_{d}\right)-\mathbf{F}\right) R(\mathbf{G}) \mathrm{d} V .
\end{aligned}
$$

Adding and subtracting $\mathbf{H}$ defined in (5.25), we get

$$
\begin{aligned}
\left\|\mathbf{p}_{d}-\mathbf{p}^{I}\right\|_{Q^{d}}^{2} & =\int_{\Omega} \mathbf{K}^{-1}\left(R\left(\mathbf{F}_{d}\right)-\mathbf{F}\right)(R(\mathbf{G})-\mathbf{H}) \mathrm{d} V+\int_{\Omega} \mathbf{K}^{-1}\left(R\left(\mathbf{F}_{d}\right)-\mathbf{F}\right) \mathbf{H} \mathrm{d} V \\
& =J_{3}+\int_{\Omega}\left(R\left(\mathbf{F}_{d}\right)-\mathbf{F}\right) \nabla \psi \mathrm{d} V=J_{3}-\int_{\Omega} \psi \operatorname{div}\left(R\left(\mathbf{F}_{d}\right)-\mathbf{F}\right) \mathrm{d} V \\
& =J_{3}-\int_{\Omega}\left(\mathbf{b}^{I}-b\right) \psi \mathrm{d} V \\
& =J_{3}-\int_{\Omega}\left(\mathbf{b}^{I}-b\right)\left(\psi-\psi^{I}\right) \mathrm{d} V=J_{3}+J_{4}
\end{aligned}
$$


In their turn, $J_{3}$ and $J_{4}$ can be easily bounded using the previous estimates and usual arguments. Indeed, the triangle inequality, then (3.10) and (5.8), and finally (5.23) and (5.34) imply that

$$
\begin{aligned}
\left\|R\left(\mathbf{F}_{d}\right)-\mathbf{F}\right\|_{\left(L_{2}(\Omega)\right)^{3}} & \leq\left\|R\left(\mathbf{F}_{d}-\mathbf{F}^{I}\right)\right\|_{\left(L_{2}(\Omega)\right)^{3}}+\left\|R\left(\mathbf{F}^{I}\right)-\mathbf{F}\right\|_{\left(L_{2}(\Omega)\right)^{3}} \\
& \leq C_{R}^{*} s_{*}^{-1 / 2}\left\|\mathbf{F}_{d}-\mathbf{F}^{I}\right\|\left\|_{X^{d}}+\right\| R\left(\mathbf{F}^{I}\right)-\mathbf{F} \|_{\left(L_{2}(\Omega)\right)^{3}} \\
& \leq C h\|p\|_{H^{2}(\Omega)} .
\end{aligned}
$$

Using the theorem assumption (5.34) and (5.24), we get

$$
\|R(\mathbf{G})-\mathbf{H}\|_{\left(L_{2}(\Omega)\right)^{3}}=\left\|R\left(\mathbf{H}^{I}\right)-\mathbf{H}\right\|_{\left(L_{2}(\Omega)\right)^{3}} \leq C_{R a}^{*} h\|\mathbf{H}\|_{\left(H^{1}(\Omega)\right)^{3}} \leq C h\left\|\mathbf{p}_{d}-\mathbf{p}^{I}\right\|_{Q^{d}} .
$$

The approximation property (5.15) gives the following estimates:

$$
\|\| \mathbf{b}^{I}-b\left\|_{L_{2}(\Omega)} \leq C_{a p p}^{*} h\right\| b \|_{H^{1}(\Omega)}
$$

and

$$
\left\|\psi-\boldsymbol{\psi}^{I}\right\|_{L_{2}(\Omega)} \leq C_{a p p}^{*} h\|\psi\|_{H^{1}(\Omega)} \leq C_{a p p}^{*} C_{\Omega}^{*} h\|\| \mathbf{p}_{d}-\mathbf{p}^{I} \|_{Q^{d}}
$$

Inserting estimates (5.37)-(5.40) into (5.36), we get immediately the result.

Remark 5.1 It is very likely that our additional assumption (5.34) is not needed, as it should be possible to deduce it from (5.4), (5.5), possibly with minor additional assumptions on the geometry. However, in essentially all cases in which $R_{E}$ can be explicitly built, it is easy to prove directly that (5.34) holds true. We decided therefore that it would have been more simple to just assume it.

\section{Conclusion}

In this paper, we have considered the mimetic finite difference method for the mixed formulation of the diffusion problem on polyhedral meshes. We have proved the stability of the mimetic discretizations and the optimal convergence rates for the scalar and vector variables. The key elements of our methodology are the consistency assumption $\mathbf{S 2}$ and the lift property $\mathbf{L P}$.

In the future work, we plan to extend the convergence results to polyhedral meshes with curvilinear faces.

\section{Acknowledgments}

The work was partly performed at the Los Alamos National Laboratory operated by the University of California for the US Department of Energy under contract W-7405-ENG-36, LAUR04-5756. The first author has been supported by FIRB 2001 and PRIN 2003 projects of Italian MIUR.

The authors thank organizers of the workshop "Compatible Spatial Discretizations for Partial Differential Equations" hold at the Institute of Mathematics and Applications (IMA) in Minneapolis for drawing attention to the problem considered in this paper: the collaboration actually started during the workshop. 


\section{Appendix A}

The purpose of this appendix is to prove the lift property (4.17)-(4.18), that we recall for convenience of the reader.

$\mathbf{L P}$ (Lift Property). For every $t<2$ there exists a constant $\lambda^{*}=\lambda^{*}(t)$ such that: For every $E \in \mathcal{T}_{h}$ and for every $e \in \partial E$ there exists a function $\varphi_{E}^{e}$ from $E$ to $\mathbb{R}$ that verifies

$$
\varphi_{E}^{e}=1 \quad \text { on } \quad e, \quad \varphi_{E}^{e}=0 \quad \text { on } \quad \partial E \backslash e,
$$

and

$$
\left\|\varphi_{E}^{e}\right\|_{L_{2}(E)} \leq \lambda^{*} h_{E}^{3 / 2}, \quad\left\|\nabla \varphi_{E}^{e}\right\|_{\left(L_{t}(E)\right)^{3}} \leq \lambda^{*} h_{E}^{3 / t-1}
$$

A traditional way would be to assume that there exist a finite number of reference elements $\hat{E}_{1}, \ldots, \hat{E}_{1}$ and a positive constant $L^{*}$ such that: For each $E \in \mathcal{T}_{h}$ there is an $\hat{E}_{k}$ and a bi-Lipschitz map $\Phi_{k}^{E}$ from $\hat{E}_{k}$ to $E$ such that

$$
\left|\Phi_{k}^{E}\right|_{W_{\infty}^{1}\left(\hat{E}_{k}\right)} \leq L^{*}, \quad\left\|\Phi_{k}^{E}\right\|_{L_{\infty}\left(\hat{E}_{k}\right)} \leq L^{*} h_{E}
$$

and

$$
\left|\left(\Phi_{k}^{E}\right)^{-1}\right|_{W_{\infty}^{1}(E)} \leq L^{*}, \quad \|\left.\left(\Phi_{k}^{E}\right)^{-1}\right|_{L_{\infty}(E)} \leq L^{*} h_{E}^{-1}
$$

Then, for each reference element $\hat{E}_{k}$ and for each face $\hat{e}$ of $\hat{E}_{k}$ we could construct the harmonic function $\hat{\varphi}_{\hat{E}_{k}}^{\hat{e}}$ with boundary value 1 on $\hat{e}$ and zero on the other faces, and verify that it belongs to $W_{t}^{1}\left(\hat{E}_{k}\right)$ for every $t<2$. Finally each function $\varphi_{E}^{e}$ could be constructed by combining one of the reference functions $\hat{\varphi}_{\hat{E}_{k}}^{\hat{e}}$ with the corresponding $\Phi_{k}^{E}$. This is surely feasible, but will become rather cumbersome if we want to consider a big variety of possible shapes for our elements.

We decided here to follow a different path, that requires only the fact that the faces are starshaped (M4) and the Pyramid property (M5) which are possibly more difficult to explain, but much easier to check and to enforce. The general idea is first to build a function $\hat{\varphi}_{1}$ on the unit cone $\mathcal{C}_{1}$, then, for every $h$, to build a function $\varphi_{h}$ on a cone $\mathcal{C}_{h}$ obtained by scaling the unit cone, and finally, for each element $E$ and for each face $e$, to map the cone $\mathcal{C}_{\gamma_{*} h_{E}}$ (where $\gamma_{*}$ is given in Assumption M4) into the pyramid $P_{E}^{e}$ described in Assumption M5 with a Lipschitz continuous mapping. This will give us a function $\varphi=\varphi_{E}^{e}$ on the pyramid, having the right norms. This function will finally be extended by zero to the whole element $E$, and it still will have the right norms. But let us see the procedure in more detail.

For each element $E$ and for each face $e$ of $E$ we want to build a function $\varphi=\varphi_{E}^{e}$ with the following properties:

- The support of $\varphi$ is contained in the pyramid $P=P_{E}^{e}$ satisfying Assumption M5.

- $\varphi \equiv 1$ on $e$ and $\varphi \equiv 0$ on the other faces of $P_{E}^{e}$.

- $\varphi$ satisfies the following estimates:

$$
\|\varphi\|_{L_{2}(P)} \leq \lambda^{*} h_{E}^{3 / 2} \quad \text { and } \quad\|\nabla \varphi\|_{\left(L_{t}(P)\right)^{3}} \leq \lambda^{*} h_{E}^{3 / t-1}
$$

where constant $\lambda^{*}$ is independent of $E$ and $e$. 
As we said before, we start our work on cones: for $\rho_{1}>0$ we shall refer to the solid

$$
\left\{(x, y, z): 0 \leq z \leq \rho_{1} \text { and } x^{2}+y^{2} \leq\left(\rho_{1}-z\right)^{2}\right\}
$$

as the circular cone of radius $\rho_{1}$.

Lemma A.1 Let $\mathcal{C}_{1}$ be the circular cone of radius 1 , and let $\hat{\varphi}_{1}$ be the harmonic function that takes value 1 on the basis and 0 on the lateral boundary. Then $\hat{\varphi}_{1}$ belongs to $L_{\infty}\left(\mathcal{C}_{1}\right)$ and $\nabla \hat{\varphi}_{1}$ belongs to $\left(L_{t}\left(\mathcal{C}_{1}\right)\right)^{3}$ for all $t<2$.

Proof. The first part of the statement follows from the maximum principle, that gives $0 \leq \hat{\varphi}_{1} \leq$ 1. The second part of the statement follows immediately from the known results concerning domains with corners (see e.g. [11], or [9]).

In view of the previous lemma, we set

$$
\hat{C}_{t}:=\left\|\nabla \varphi_{1}\right\|_{\left(L_{t}\left(\mathcal{C}_{1}\right)\right)^{3}} .
$$

It is clear that $\hat{C}_{t}$ depends on $t$ and hence on $s$ through (4.15).

Lemma A.2 For every positive real number $h$, let $\mathcal{C}_{h}$ be a circular cone of radius $h$. Then, there exists a function $\varphi_{h}$ taking value 1 on the basis, value zero on the lateral surface, and satisfying

$$
\|\left.\varphi_{h}\right|_{L_{2}\left(\mathcal{C}_{h}\right)} \leq\left|\mathcal{C}_{h}\right|^{1 / 2} \quad \text { and } \quad\left\|\nabla \varphi_{h}\right\|_{\left(L_{t}\left(\mathcal{C}_{h}\right)\right)^{3}} \leq h^{3 / t-1} \hat{C}_{t}
$$

where $\left|\mathcal{C}_{h}\right|$ is the volume of $\mathcal{C}_{h}$.

The proof follows with the usual scaling arguments (see e.g. [8, Theorem 3.1.2]).

Consider now a face $e$ of $E$. For convenience, we assume that (a) the face $e$ lies in the plane $z=0$, (b) $M_{e}$, defined in Assumption M4 (Star-shaped faces), is the origin of the axes and (c) the polyhedron $E$ is locally in the half-space $z>0$. By Assumption M4 there exists a $\gamma_{*}$ such that: The circular cone $\mathcal{C}_{h}$ having the basis on the face $e$ (with center in $M_{e}$ ), and radius $h=\rho_{*}=\gamma_{*} h_{E}$, is strictly contained in the pyramid $P_{E}^{e}$ having the same vertex and basis equal to $e$. Hence, by Assumption M5 (The pyramid property), $\mathcal{C}_{h}$ is contained in $E$.

Let us see first that Assumption M4 implies the existence of a radial mapping in the plane $z=0$ which maps the disk $D_{\rho_{*}}$ with center in $M_{e}$ and radius $\rho_{*}$ into the face $e$, is one-to-one, Lipschitz-continuous together with its inverse, and with $W_{\infty}^{1}$ norms bounded in terms of $\gamma_{*}$ and the number of edges of $e$.

Lemma A.3 Under assumption M4 there exists a map $\Phi_{2}$, mapping the disk $D_{\rho_{*}}$ into the face $e$, that is Lipschitz continuous together with the inverse map $\Phi_{2}^{-1}$. Moreover,

$$
\left\|\Phi_{2}\right\|_{W_{\infty}^{1}\left(D_{\rho_{*}}\right)} \leq C_{e}^{*} \quad \text { and } \quad\left\|\Phi_{2}^{-1}\right\|_{W_{\infty}^{1}(e)} \leq C_{e}^{*}
$$

where $C_{e}^{*}$ depends only on the constant $\gamma_{*}$ of Assumption M4. 


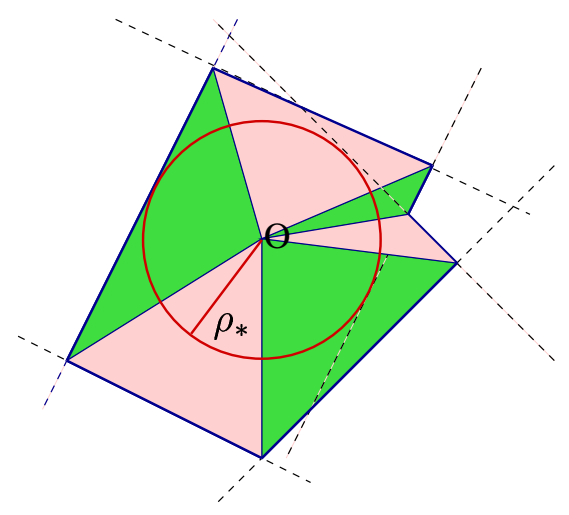

Figure 3: The splitting of $e$ in sectors.

Proof. To show this, we note that the plane $z=0$ can be split in a finite number of sectors by the vertices of $e$. Each sector corresponds to the straight rays coming out of the origin $M_{e}$ and intersecting the edge $\ell_{k}$ (see Fig. 3). For each point $\mathbf{P} \in D_{\rho_{*}}$, we first consider the ray emanating from the origin and passing through $\mathbf{P}$. This ray intersects $\partial e$ at point $\mathbf{V}(\mathbf{P})$. Our mapping is defined as follows:

$$
\tilde{\mathbf{P}} \equiv \Phi_{2}(\mathbf{P}):=\frac{|\mathbf{V}(\mathbf{P})|}{\rho_{*}} \mathbf{P} .
$$

It is clear that $\Phi_{2}$ maps every point $\mathbf{P}$ into a point $\tilde{\mathbf{P}}$ on the same ray, so that

$$
\mathbf{V}(\mathbf{P})=\mathbf{V}(\tilde{\mathbf{P}}) \quad \forall \mathbf{P} \in D_{\rho^{*}}
$$

It is immediate to check that, on each ray, the map is continuous and monotone, and that it maps the points of the circumference of radius $\rho_{*}$ into the corresponding points of $\partial e$ on the same ray. Hence it maps $D_{\rho_{*}}$ into $e$ in a one-to-one way. It is also clear that the map is globally continuous, invertible, and the inverse map

$$
\mathbf{P} \equiv \Phi_{2}^{-1}(\tilde{\mathbf{P}}):=\frac{\rho_{*}}{|\mathbf{V}(\mathbf{P})|} \tilde{\mathbf{P}} \equiv \frac{\rho_{*}}{|\mathbf{V}(\tilde{\mathbf{P}})|} \tilde{\mathbf{P}}
$$

is also continuous and maps $e$ into $D_{\rho_{*}}$. Note that we used (A.10) in the last step.

In order to show the Lipschitz continuity, we have to bound the distance between the images $|\tilde{\mathbf{P}}-\tilde{\mathbf{Q}}|$ by a constant times the distance $|\mathbf{P}-\mathbf{Q}|$. For this, we remark first that Assumption M4 implies that

$$
1 \leq \frac{|\mathbf{V}|}{\rho_{*}} \leq \frac{h_{E}}{\gamma_{*} h_{E}}=\frac{1}{\gamma_{*}} \quad \text { for every } \mathbf{V} \in \partial e .
$$

As shown in Figure 4, it also implies that for every point $\mathbf{V}$ on an edge $\ell$ of $\partial e$, the angle $\alpha_{V}$ between $\ell$ and the ray passing through $\mathbf{V}$ verifies

$$
\left|\sin \alpha_{V}\right|=\frac{\left|\mathbf{H}_{\ell}\right|}{|\mathbf{V}|} \geq \frac{\rho_{*}}{|\mathbf{V}|} \geq \gamma_{*}
$$

where $\mathbf{H}_{\ell}$ is the orthogonal projection of the origin $M_{e}$ on the line containing $\ell$, and we used (A.12) in the last step. 


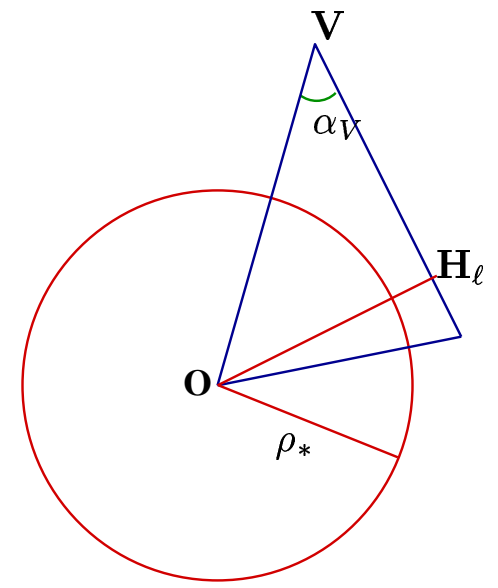

Figure 4: Lower bound on $\left|\sin \alpha_{V}\right|$.

The Lipschitz continuity is obvious when $\mathbf{P}$ and $\mathbf{Q}$ are on the same ray:

$$
|\tilde{\mathbf{P}}-\tilde{\mathbf{Q}}|=\frac{|\mathbf{V}(\mathbf{P})|}{\rho_{*}}|\mathbf{P}-\mathbf{Q}| \leq \frac{1}{\gamma_{*}}|\mathbf{P}-\mathbf{Q}| \text {. }
$$

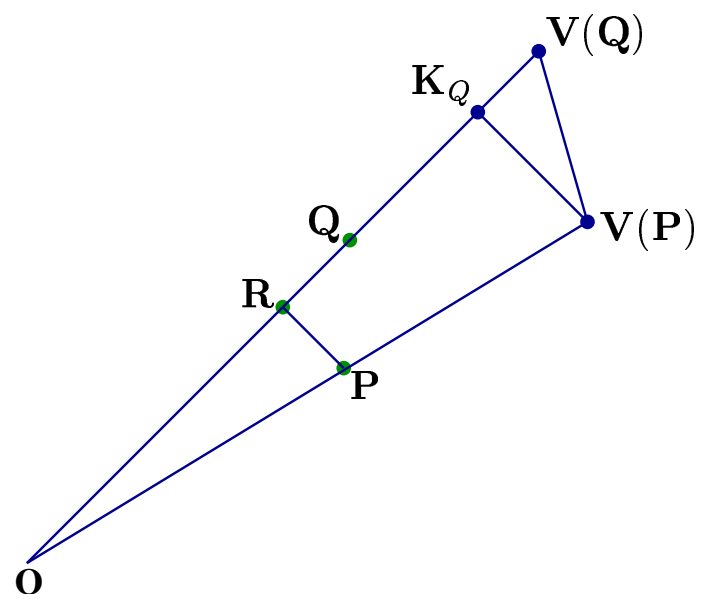

Figure 5: Lipschitz continuity within a sector.

If $\mathbf{P}$ and $\mathbf{Q}$ are on two different rays in the same sector, we first denote by $\mathbf{K}_{Q}$ and $\mathbf{R}$ (respectively) the orthogonal projections of $\mathbf{V}(\mathbf{P})$ (respectively, of $\mathbf{P}$ ) on the ray containing $\mathbf{Q}$ (see Figure 5). Applying Thaletes theorem, we get

$$
\frac{\left|\mathbf{V}(\mathbf{P})-\mathbf{K}_{Q}\right|}{|\mathbf{V}(\mathbf{P})|}=\frac{|\mathbf{P}-\mathbf{R}|}{|\mathbf{P}|} \leq \frac{|\mathbf{P}-\mathbf{Q}|}{|\mathbf{P}|}
$$

Collecting (A.15), (A.13) and (A.12), we have

$$
|\mathbf{V}(\mathbf{P})-\mathbf{V}(\mathbf{Q})|=\frac{\left|\mathbf{V}(\mathbf{P})-\mathbf{K}_{Q}\right|}{\left|\sin \left(\alpha_{V(Q)}\right)\right|} \leq \frac{|\mathbf{P}-\mathbf{Q}|}{\gamma_{*}|\mathbf{P}|}|\mathbf{V}(\mathbf{P})| \leq \frac{|\mathbf{P}-\mathbf{Q}|}{\left(\gamma_{*}\right)^{2}|\mathbf{P}|} \rho_{*}
$$


where obviously the role of $\mathbf{P}$ and $\mathbf{Q}$ can be interchanged. Finally, the triangle inequality together with (A.9) and (A.16) give

$$
\begin{aligned}
|\tilde{\mathbf{P}}-\tilde{\mathbf{Q}}| & =\left|\frac{|\mathbf{V}(\mathbf{P})| \mathbf{P}-|\mathbf{V}(\mathbf{Q})| \mathbf{Q}}{\rho_{*}}\right| \leq \frac{|\mathbf{V}(\mathbf{P})-\mathbf{V}(\mathbf{Q})|}{\rho_{*}}|\mathbf{P}|+\frac{|\mathbf{V}(\mathbf{Q})|}{\rho_{*}}|\mathbf{P}-\mathbf{Q}| \\
& \leq \frac{|\mathbf{P}-\mathbf{Q}|}{\left(\gamma_{*}\right)^{2}}+\frac{1}{\gamma_{*}}|\mathbf{P}-\mathbf{Q}|=\frac{1+\gamma_{*}}{\left(\gamma_{*}\right)^{2}}|\mathbf{P}-\mathbf{Q}| .
\end{aligned}
$$

The case of $\mathbf{P}$ and $\mathbf{Q}$ belonging to different sectors can be easily deduced inserting suitable intermediate points at the boundaries of the sectors and then using the triangle inequality.

In a similar way, we can show that the inverse mapping is also Lipschitz continuous. For instance, using (A.11) we get

$$
|\mathbf{P}-\mathbf{Q}|=\left|\frac{\rho_{*}}{|\mathbf{V}(\tilde{\mathbf{P}})|} \tilde{\mathbf{P}}-\frac{\rho_{*}}{|\mathbf{V}(\tilde{\mathbf{Q}})|} \tilde{\mathbf{Q}}\right|=\frac{\rho_{*}}{|\mathbf{V}(\tilde{\mathbf{P}})||\mathbf{V}(\tilde{\mathbf{Q}})|}|| \mathbf{V}(\tilde{\mathbf{Q}})|\tilde{\mathbf{P}}-| \mathbf{V}(\tilde{\mathbf{P}})|\tilde{\mathbf{Q}}| .
$$

Then, we have, adding and subtracting $|\mathbf{V}(\mathbf{P})| \mathbf{P}$ and using the triangle inequality,

$$
|| \mathbf{V}(\tilde{\mathbf{Q}})|\tilde{\mathbf{P}}-| \mathbf{V}(\tilde{\mathbf{P}})|\tilde{\mathbf{Q}}| \leq|\mathbf{V}(\tilde{\mathbf{P}})-\mathbf{V}(\tilde{\mathbf{Q}})||\tilde{\mathbf{P}}|+|\mathbf{V}(\tilde{\mathbf{P}})||\tilde{\mathbf{P}}-\tilde{\mathbf{Q}}| .
$$

On the other hand, we can apply the argument of (A.16) to obtain

$$
|\mathbf{V}(\tilde{\mathbf{P}})-\mathbf{V}(\tilde{\mathbf{Q}})| \leq \frac{|\tilde{\mathbf{P}}-\tilde{\mathbf{Q}}|}{\left(\gamma_{*}\right)^{2}|\tilde{\mathbf{P}}|} \rho_{*}
$$

Collecting (A.18), (A.19) and (A.20), and using (A.12) (this time in the sense $\rho_{*} /|\mathbf{V}| \leq 1$ ), we finally obtain

$$
|\mathbf{P}-\mathbf{Q}| \leq \frac{1}{\left(\gamma_{*}\right)^{2}}|\tilde{\mathbf{P}}-\tilde{\mathbf{Q}}|+|\tilde{\mathbf{P}}-\tilde{\mathbf{Q}}|=\frac{1+\left(\gamma_{*}\right)^{2}}{\left(\gamma_{*}\right)^{2}}|\tilde{\mathbf{P}}-\tilde{\mathbf{Q}}| .
$$

This proves the assertion of the lemma.

Now, we can construct a mapping $\Phi_{3}$ from the cone $\mathcal{C}_{h}$ (having $D_{\rho_{*}}$ as basis and with height equal to $\rho_{*}$ ) into to the pyramid $P_{E}^{e}$ (having $e$ as basis and with the same vertex as $\mathcal{C}_{h}$ ), also Lipschitz-continuous with its inverse, by taking

$$
(\tilde{x}, \tilde{y})=\Phi_{2}(x, y), \quad \tilde{z}=z .
$$

Again, the Lipschitz norm of the map $\Phi_{3}$ and of its inverse depend only on $\gamma_{*}$. This proves the following lemma.

Lemma A.4 Under assumption M4 there exists a map $\Phi_{3}$, mapping the cone $\mathcal{C}_{h}$ into the pyramid $P_{E}^{e}$, that is Lipschitz continuous together with the inverse map $\Phi_{3}^{-1}$. Moreover,

$$
\left\|\Phi_{3}\right\|_{W_{\infty}^{1}\left(\mathcal{C}_{h}\right)} \leq C_{p y r}^{*} \quad \text { and } \quad\left\|\Phi_{3}^{-1}\right\|_{W_{\infty}^{1}\left(P_{E}^{e}\right)} \leq C_{p y r}^{*}
$$

where $C_{p y r}^{*}$ depends only on the constant $\gamma_{*}$ of Assumption M4. 
The last step is to construct, for each element $E$ and for each face $e \in \partial E$, the function $\varphi_{E}^{e}$ satisfying (A.5) (with the right boundary conditions). Let

$$
\varphi_{E}^{e}(x, y, z)=\varphi_{h}\left(\Phi_{3}^{-1}(x, y, z)\right)
$$

where $\varphi_{h}$ is the function from Lemma A.2 defined for the circular cone of radius $h=\rho_{*}=$ $\gamma_{*} h_{E}$. It is clear that $\varphi_{E}^{e}$ will be in $L_{2}\left(P_{E}^{e}\right)$, that $\nabla \varphi_{E}^{e}$ will be in $\left(L_{t}\left(P_{E}^{e}\right)\right)^{3}$, and that their norms will be bounded by

$$
\left\|\varphi_{E}^{e}\right\|_{L_{2}\left(P_{E}^{e}\right)} \leq C_{p y r}^{*} h_{E}^{3 / 2} \quad \text { and } \quad\left\|\nabla \varphi_{E}^{e}\right\|_{\left(L_{t}\left(P_{E}^{e}\right)\right)^{3}} \leq \hat{C}_{t} C_{p y r}^{*} h_{E}^{3 / t-1}
$$

where $\hat{C}_{t}$ is given in (A.6) and $C_{p y r}^{*}$ depends only on $\gamma_{*}$. Hence $\varphi_{E}^{e}$ satisfies (A.5) as required. Finally, we take the prolongation of $\varphi_{E}^{e}$ (that we call again $\varphi_{E}^{e}$ ) by zero in $E \backslash P_{E}^{e}$.

This ends the proof of the lift property (A.1)-(A.2).

\section{References}

[1] D. Arnold. An interior penalty finite element method with discontinuous elements. SIAM J. Numer. Anal., 19:742-760, 1982.

[2] M. Berndt, K. Lipnikov, J. D. Moulton, and M. Shashkov. Convergence of mimetic finite difference discretizations of the diffusion equation. East-West J. Numer. Math., 9:253-284, 2001.

[3] M. Berndt, K. Lipnikov, M. Shashkov, M. Wheeler, and I. Yotov. Superconvergence of the velocity in mimetic finite difference methods on quadrilaterals. Submitted to Numerische Mathematik, 2004.

[4] S. Brenner and L. Scott. The mathematical theory of finite element methods. SpringerVerlag, Berlin/Heidelberg, 1994.

[5] F. Brezzi and M. Fortin. Mixed and hybrid finite element methods. Springer-Verlag, New York, 1991.

[6] D. Burton. Multidimensional discretization of conservation laws for unstructured polyhedral grids. Technical Report UCRL-JC-118306, Lawrence Livermore National Laboratory, 1994.

[7] J. Campbell and M. Shashkov. A tensor artificial viscosity using a mimetic finite difference algorithm. J. Comput. Phys., 172:739-765, 2001.

[8] P.G. Ciarlet. The finite element method for elliptic problems. North-Holland, New York, 1978.

[9] M. Dauge. Elliptic boundary value problems on corner domains: smoothness and asymptotics of solutions. Springer-Verlag, Berlin, New York, 1988.

[10] J. Douglas and J. Roberts. Global estimates for mixed methods for second order elliptic equations. Mathematics of Computations, 44(169):39-52, 1985. 
[11] P. Grisvard. Elliptic Problems in Nonsmooth domains. Pitman, London, 1985.

[12] J. Hyman, J. Morel, M. Shashkov, and S. Steinberg. Mimetic finite difference methods for diffusion equations. Comp. Geosciences, 6(3-4):333-352, 2002.

[13] J. Hyman and M. Shashkov. Mimetic discretizations for Maxwell's equations and the equations of magnetic diffusion. Progress in Electromagnetic Research, 32:89-121, 2001.

[14] J. Hyman, M. Shashkov, and S. Steinberg. The numerical solution of diffusion problems in strongly heterogeneous non-isotropic materials. J. Comput. Phys., 132:130-148, 1997.

[15] Y. Kuznetsov, K. Lipnikov, and M. Shashkov. Mimetic finite difference method on polygonal meshes for diffusion-type problems. Technical Report LAUR-03-7608, Los Alamos National Laboratory, 2003. Submitted to Comp. Geosciences.

[16] K. Lipnikov, J. Morel, and M. Shashkov. Mimetic finite difference methods for diffusion equations on non-orthogonal non-conformal meshes. J. Comput. Phys., (in press), 2004.

[17] R. Liska, M. Shashkov, and V. Ganza. Analysis and optimization of inner products for mimetic finite difference methods on triangular grid. Mathematics and Computers in Simulation, 67:55-66, 2004.

[18] L. Margolin, M. Shashkov, and P. Smolarkiewicz. A discrete operator calculus for finite difference approximations. Comput. Meth. Appl. Mech. Engrg., 187:365-383, 2000.

[19] J. Morel, R. Roberts, and M. Shashkov. A local support-operators diffusion discretization scheme for quadrilateral $r-z$ meshes. J. Comput. Phys., 144:17-51, 1998.

[20] T. Palmer, M. Zika, and N. Madsen. Unstructured polyhedral mesh thermal radiation diffusion. Transactions of the American Nuclear Society, 83:248-9, 2000.

[21] K. Thompson and M. Adams. A spatial discretization for solving the transport equation on unstructured grids of polyhedra. In Proc. International Conference on Mathematics and Computation, Reactor Physics and Environmental Analysis in Nuclear Applications, volume II, pages 1196-1204, 1994. 